\title{
L'ACCORD DE PAIX SUR LA BOSNIE-HERZÉGOVINE DU 14 DÉCEMBRE 1995 : UN TRAITÉ SOUS BÉNÉFICE D'INVENTAIRE
}

\author{
JEAN-MARC SOREL
}

L'accord de paix sur la Bosnie-Herzégovine, paraphé à Dayton (EtatsUnis) le 21 novembre 1995 et signé à Paris le 14 décembre $1995(1)$, vient clore, d'une manière que l'on espère définitive, le plus long et le plus sanglant conflit en Europe depuis la deuxième guerre mondiale.

Il faudrait sans doute le talent de G. Bernanos (celui des Grands cimetières sous la lune) pour rédiger l'ouvrage définitif sur un tel conflit. Celui-ci serait d'ailleurs prématuré car si le traité de Paris permet une solution pour le moment satisfaisante, rien n'indique, à l'heure actuelle, que la stabilisation de la région soit acquise (2). Depuis 1991, la guerre a ravagé l'ex-Yougoslavie, avec une intensité particulière en Bosnie-Herzégovine, et ce conflit a connu de nombreuses phases dans les tentatives de règlement par des Etats, des regroupements d'Etats ou des organisations internationales. On retrouve la trace de toutes ces entités dans le traité de Paris, et c'est pourquoi il est nécessaire de revenir rapidement sur le contexte de l'accord de paix (ceci sans prétendre à une quelconque exhaustivité(3)), avant d'envisager ses principales dispositions.

(*) Jean-Marc SOREL, professeur à l'Université de Rennes I.

(1) L'accord du 14 décembre 1995, signé au palais de l'Elysée, est souvent mentionné (notamment dans la presse) sous l'expression * accord(s) de Dayton *. Cette assimilation provient de la confusion entre l'authentification et le consentement à être lié, c'est-à-dire entre le texte du traité de paix qui fut négocié et approuvé (paraphé) lors des * pourparlers de paix de proximité * sur la base aérienne de Wright-Patterson a Dayton (Ohio) du $1^{\text {er }}$ au 21 novembre 1995, et le traité définitivement accepté par la signature des parties à Paris (au Palais de l'Elysée) le 14 décembre 1995 . Il convient de signaler également que seule la version en langue anglaise du traité fait foi. Nous avons, par conséquent, utilisé la traduction provisoire du traité fournie par le ministère des affaires étrangères pour les citations mais nous mentionnons, lorsqu'un doute surgit sur un mot ou une expression, la version originale entre parenthèses.

(2) Il est utile d'indiquer que les quelques remarques qui vont suivre ont été rédigées en février-mars 1996 et que la date symbolique de la réunification de Sarajevo, le 19 mars 1996, est celle au-delà de laquelle les faits nouveaux en rapport avec le processus de paix ne sont plus pris en compte.

(3) La littérature politique et juridique sur ce conflit est très importante. Parmi celle-ci, nous pouvons mentionner: X. Bovghez: : * Bosnie-Herzégovine, anatomie d'une poudrière *, Hérodote $\mathrm{n}^{\circ} 67$, octobre-décembre 1992 (dossier sur la question serbe); M. WELLER : "The International Response to the Dissolution of the Socialist. Federal Republic of Yougoslavia *, AJIL, vol. 86, 1992, pp. 569-607; D. Petrovic et L. Condorella : $\propto$ L'ONU et la crise yougoslave s, AFDI, 1992, pp. $32-$ 60 ; D. Vernet et Jean-Marc GoNTN : Rêve sacrifté, chronique des guerres yougoslaves, éd. Odile Jacob, Paris, 1994 ; J. RUPNIK (dir.) : Le déchirement des nations, Seuil-CERI (collection * l'idée du monde n), Paris, 1995, 283 p. (voir : S. Pлvlowiтch : "Yougoslavie : de l'idéal d'un Etat-nation à la barbarie des pouvoirs ethniques "); Général Cor (dir.): Opérations des Nations Unies, leçons de 


\section{INTRODUCTION : LE CONTEXTE DE L'ACCORD DE PAIX}

La Yougoslavie constituait un Etat fédéral particulier par la diversité de ses composantes territoriales, ethniques ou religieuses. La Bosnie-Herzégovine était, parmi les Etats fédérés, une illustration de cette diversité puisqu'y coexistaient des musulmans, des serbes et des croates, sans que ces dénominations recouvrent une distinction claire, notamment du point de vue religieux (4). L'histoire très complexe de la formation de l'Etat yougoslave permet de comprendre, qu'au-delà des querelles ethniques ou religieuses, c'est un ensemble de facteurs qui opposent les différentes composantes de l'ex-Yougoslavie dont la Bosnie-Herzégovine est un microcosme. On note, par exemple, une coïncidence plus nette entre l'Etat et la nation croates (qui fut un Etat indépendant de 1941 à 1945 s'illustrant par des massacres ethniques, et notamment serbes, sous la férule d'Ante Pavelic), alors que le "territoire" serbe correspond davantage à une vision ethnique et communautaire, ce qui introduit un élément de variabilité très dangereux. Dans ce contexte, l'affirmation d'une identité de la Bosnie-Herzégovine comme Etat-nation se fera à partir de 1991 "par défaut"(5). Désormais, les $51 \%$ attribués à la Fédération croato-musulmane et les $49 \%$ attribués à la République serbe de Bosnie figent le cadre de cet Etat dont la variété de sa population aurait pu être une chance et une richesse et fut, à l'inverse, la cause de son anéantissement.

La proclamation de l'indépendance de la Slovénie et de la Croatie le 25 juin 1991 marque le début du conflit dans l'ex-Yougoslavie. La proclamation de l'indépendance de la Bosnie-Herzégovine n'interviendra que le 3 mars 1992 à la suite d'un référendum le $1^{\text {er }}$ mars qui l'approuve à une majorité de $63 \%$. Cet Etat sera rapidement reconnu par la Communauté européenne le 6 avril, puis par les Etats-Unis le 7 avril alors que se crée parallelement un nouvel Etat yougoslave, le 27 avril, formé par la Serbie et le Monténégro. Ce mouvement rapide d'indépendances fut largement soutenu par une attitude compréhensive des Etats occidentaux. L'impulsion provient de l'avis $\mathrm{n}^{\circ} 1$ de la Commission d'arbitrage pour la paix dans l'ex-Yougoslavie du 29 novembre 1991 reconnaissant «que la République Socialiste Féderative

\footnotetext{
(suite note 3 )

terrain (Cambodge, Somalie, Rwanda, Yougoslavie), Fondation pour les Etudes de Defense (collection "Perspectives stratégiques"), Paris, 1995, 390 p.; Z. Dizdarevic et G. Riva : J'accuse l'ONU, Calmann-Levy, Paris, 1995, 207 p.; P. GARDE : Journal de voyage en Bosnie-Herzégovine, éd. La Nuée Bleue, Paris, 1995, 141 p. ; A. Fontaine : Après eux, le déluge (de Kaboul à Sarajevo), Fayard, Paris, 1995, 658 p. (voir le passage sur la Yougoslavie, pp. 596-618); J. Julliard : Pour la Bosnie, Seuil, Paris, 1995, 320 p.; E. MoRIN : Les fratricides, Yougoslavie 1991-1995, Arléa, Paris, 1995, 124 p.; P. HASSNER : "Ex-Yougoslavie: le tournant?", Politique internationale, n" 69, automne 1995 , pp. 208 et $\mathrm{s}$. Ces ouvrages abordent la question de l'ex-Yougoslavie sous divers angles mais constituent tous des témoignages précieux. Il est évident que ce conflit ne peut être envisagé d'un point de vue uniquement juridique, la compréhension de l'ensemble des problèmes est, par conséquent, indispensable. Nous ne saurions trop recommander pour conclure cette liste bibliographique la vision du film d'Emir Kusturica (Underground). Cette référence iconoclaste est précieuse pour comprendre le regret par certains (dont le réalisateur) d'un pays qui s'appelait la Yougoslavie.

(4) X. Bougarel (ibid.) remarque, qu'en 1990 , il y avait environ $44 \%$ de musulmans (dont $16,5 \%$ au sens religieux), $31 \%$ de serbes (dont $20 \%$ d'orthodoxes) et $17,3 \%$ de croates (dont $15 \%$ de catholiques). La proportion non négligeable de $46 \%$ se déclarait sans confession.

(5) Voir : J. KRULIC « La perception de l'Etat-nation par les croates, les musulmans bosniaques et les serbes ", dans : Nations et nationalismes, éd. La découverte, Paris, 1995, pp. 108-113.
} 
de Yougoslavie est engagée dans un processus de dissolution»(6). Dès lors, la Communauté européenne engage le processus de reconnaissance des nouvelles Républiques en l'assortissant de conditions (7), alors que la Commission d'arbitrage, par une série d'avis du 11 janvier 1991, admet que les frontières des anciennes Républiques fédérées deviennent des frontières internationales (en respectant le principe de l'uti possidetis), donne un avis positif à la reconnaissance de la Slovénie et de la Macédoine, demande des modifications constitutionnelles pour la reconnaissance de la Croatie et rejette la reconnaissance de la République socialiste de Bosnie-Herzégovine (proclamée une première fois le 15 octobre 1991) car elle ne concerne pas tous les citoyens de cette République, les serbes ayant réclamé un "Etat yougoslave commun" (le 10 novembre 1991), puis ayant proclamé une "République serbe de Bosnie-Herzégovine" (le 21 décembre 1992)(8). L'ONU, par la voie du Conseil de sécurité, est également entrée en action depuis le 29 septembre 1991 lors de l'adoption de la résolution 713 qui impose un embargo sur les livraisons d'armements et les équipements militaires, mais le Conseil de sécurité confie le règlement politique du conflit à la Communauté européenne et à la CSCE via le chapitre VIII de la Charte. L'idée d'une force de maintien de la paix suivra son cours jusqu'à la création de la FORPRONU par la résolution 743 du 21 février 1992. En avril, c'est le début de l'encerclement de Sarajevo par les forces serbes.

A partir de ces prémices où les principaux acteurs sont déjà présents (Etats et organisations internationales), le conflit va perdurer jusqu'en 1995 au rythme de cessez-le-feu fréquents mais jamais respectés et de plans de paix toujours rejetés (six plans vont se succéder avant celui de Dayton). Les principales phases sont connues mais méritent un rapide rappel pour comprendre la complexité des interventions lors de l'accord de paix.

Le 11 décembre 1992 par la résolution 795, le Conseil de sécurité autorise pour la première fois le déploiement préventif des casques bleus en Macédoine à la demande du gouvernement. Progressivement, la Serbie et le Monténégro sont mis au ban de la société internationale, notamment par leur «exclusion" plus ou moins déguisée d'organisations internationales : le 15 décembre 1992, la Yougoslavie n'existe plus aux yeux du FMI et le 19 septembre 1993, la résolution $777 \mathrm{du}$ conseil de sécurité "considère que la République Fédérative de Yougoslavie (Serbie et Monténégro) ne peut pas assurer automatiquement la continuité de la qualité de membre de l'ancienne République Fédérative Socialiste de Yougoslavie aux Nations Unies et par conséquent recommande à l'Assemblée générale de décider que la République Fédérative de Yougoslavie (Serbie et Monténégro) devrait présenter une demande d'adhésion aux Nations Unies et qu'elle ne participera pas aux travaux de l'Assemblée générale". Néanmoins, peu à peu, les conférences entérinent les victoires des agresseurs. La Conférence sur la Yougoslavie

(6) Texte dans la RGDIP 1992/1, pp. 264-266.

(7) La Déclaration sur la Yougoslavie du 16 décembre 1991 demande que les nouvelles Républiques acceptent les "engagements contenus dans les lignes directrices" adoptées le même jour, à Bruxelles, et qui concernent aussi bien l'ex-Union soviétique que les nouveaux Etats en Europe orientale (textes dans RGDIP 1992/1, pp. 261 à 263). Outre l'assurance que les Etats membres ne reconnaîtront pas des entités qui seraient le résultat d'une agression, les lignes directrices rappellent l'inviolabilité des limites territoriales et demandent : "La garantie des droits des groupes ethniques et nationaux et des minorités conformément aux engagements souscrits dans le cadre de la CSCE ". A la lumière de l'accord de Paris, ces textes paraissent très lointains.

(8) Avis $\mathrm{n}^{\circ} 3$ (texte dans RGDIP 1992/1 pp. 267-269) et $\mathrm{n}^{\circ} 4$ à $\mathrm{n}^{\circ} 7$ (textes dans RGDIP $1993 / 2$, pp. 565 à 583 ). 
instituée par les Communautés européennes en vertu de la Déclaration ministérielle du 27 août 1991 et présidée par Lord Carrington avait pour mission de chercher une solution unitaire en liaison avec la Commission d'arbitrage présidée par R. Badinter mais les plans proposés (notamment le plan Vance-Owen) optent pour une cantonisation de la Bosnie entre musulmans, croates et serbes tout en essayant de maintenir la fiction d'un cadre constitutionnel unitaire. C'est la préfiguration des accords actuels.

Parallc̀lement, l'OTAN et l'UEO constituent un commandement unifié en adriatique le 15 juin 1993, sous le contrôle de l'ONU, dans le cadre de la mission Sharp Guard d'embargo sur l'ex-Yougoslavie. Le 9 août 1993, le Conseil de l'Atlantique Nord (qui regroupe les ambassadeurs des seize pays membres auprès de l'OTAN) adopte le principe "d'options opérationnelles" en Bosnie. Il s'agit de frappes aériennes pour assurer la sécurité des personnels de la FORPRONU et dessérer l'étau serbe autour de Sarajevo. C'est la première fois depuis sa création en 1949 que l'OTAN intervient militairement mais ces actions ne doivent pas être considérées comme la volonté d'intervenir militairement dans le conflit. Début 1994, les imperfections de la chaîne de commandement des casques bleus en ex-Yougoslavie sont évidentes, même si juridiquement la situation est clarifiée par les résolutions 807 du 19 février 1993 (qui autorise le recours à la force pour la sécurité des casques bleus), 816 du 31 mars 1993 (qui autorise les bombardements aériens pour le respect des zones d'exclusion aériennes) et 836 du 4 juin 1993 (qui autorise les bombardements aériens pour la protection des «zones de sécurité" qui viennent d'être créées par la résolution 824 du 6 mai $1993(9)$ ). A la suite d'un obus meurtrier tombé sur le marché de Sarajevo, le Conseil de l'Atlantique Nord décide d'autoriser le recours à des frappes aériennes pour soutenir l'action de la FORPRONU à Sarajevo(10). Néanmoins, cette décision fut jugée trop tardive pour redresser une situation que la FORPRONU ne contrôlait plus guère. Les généraux se succèdent à la tête de la FORPRONU, les résolutions se multiplient (plus de cinquante sur l'ex-Yougoslavie), les casques bleus sont toujours plus nombreux (46 000 hommes de 35 pays au plus fort de la présence de la FORPRONU) mais la situation militaire évolue à la faveur d'un pur rapport de forces favorable aux serbes de Bosnie.

La Cour internationale de Justice est également saisie dans le cadre de mesures conservatoires demandées par la Bosnie. Après les mesures prononcées par l'ordonnance du 8 avril 1993, la Bosnie n'obtient pas de nouvelles mesures par l'ordonnance du 13 septembre 1993, la Cour se contentant de réitérer les mesures précédentes, par ailleurs inappliquées. La création du Tribunal Pénal International pour les crimes commis dans l'ex-Yougoslavie par la résolution 808 du 22 février 1993 est presque considérée, par les responsables politiques, comme un aléa sans suite sérieuse. A ce moment, les serbes de Bosnie contrôlent $70 \%$ du territoire de la Bosnie alors que le plan Owen-Stoltenberg d'août 1993 leur en accordait $52 \%$.

(9) Il s'agit des six zones de sécurité dont le sort illustre tristement l'impuissance des Nations Unies face à leurs propres engagements: Sarajevo, Tuzla (qui fut bombardée par les serbes le 25 mai 1995), Zepa, Goradze, Bihac et Srebrenica (prise par les serbes le 10 juillet 1995 et donnant lieu à un véritable génocide de la population). A ces villes martyrs, il faudrait ajouter les noms de Vukovar (symbole de la résistance croate qui tombe le 18 novembre 1991), Mostar (où une guerre dans la guerre éclata en mars 1993 entre les croates et les musulmans) ou encore : Prijeda, Keraterm et Omarska qui furent des camps serbes de prisonniers.

(10) Décision du 9 février 1994, texte dans RGDIP 1994/2, pp. 567-568. 
Un des rares éléments positifs réside dans l'accord imposé par les Etats-Unis aux croates et aux musulmans le $1^{\text {er }}$ mars 1994 à Washington qui crée une Fédération croato-musulmane (elle-même autorisée à se "confédérer" avec la Croatie). Cette Fédération entérinée le 13 mars suivant, à Vienne, par les principaux intéressés ne fonctionnera guère mais sera à la base de la construction territoriale et institutionnelle de l'accord de paix.

L'année 1995 ne s'ouvrait pas sur une note optimiste, la lassitude de solutions toujours avortées ayant largement découragé les multiples intervenants. L'année commence par l'échec d'un nouveau cessez-le-feu et la modification des mandats des casques bleus (11) mais l'équilibre militaire se modifie à partir du mois de mai avec la défaite des milices serbes face à l'armée croate en Krajina, cette région aux "confins» de la Croatie et de la Serbie qui fut autrefois la limite entre l'empire austro-hongrois et l'empire ottoman. Après l'humiliation de la prise en otage de près de 400 casques bleus en représailles de bombardements de l'OTAN, le Conseil de sécurité décide de créer, à l'initiative de la France, une Force multinationale de Réaction Rapide (FRR) qui doit appuyer la FORPRONU en Bosnie. Cette nouvelle force de 4000 hommes est décidée le 3 juin 1995 entre l'OTAN et l'UEO et entérinée par la résolution 998 du 16 juin 1995(12). La nouvelle donne militaire incite les Etats-Unis à mettre en pratique une mesure qu'ils souhaitaient depuis longtemps : le 26 juin 1995 le Sénat américain vote la levée unilatérale de l'embargo sur les armes au profit de la Bosnie (celui-ci était en vigueur depuis 1991) «au nom du droit des bosniaques à se défendre eux-mêmes ", et le 13 août, les Etats-Unis lancent une initiative diplomatique s'inspirant du plan du Groupe de Contact. Cette initiative aboutira aux négociations de Dayton. Il est vrai qu'en septembre 1995, différentes offensives militaires jointes à la pression de la FRR ont permis aux bosniaques de reconquérir $20 \%$ du territoire occupé par les serbes de Bosnie, ce nouvel équilibre proche du découpage proposé par les différents plans de paix permettant l'amorce d'une négociation.

Le bilan de ce long conflit est éloquent(13) et les enseignements que l'on pourra en tirer sont nombreux. Il n'y a, à proprement parler, aucun vainqueur dans cette guerre et la communauté internationale a plus montré ses faiblesses que sa force collective. Faute d'entente politique, l'Union européenne s'est rapidement cantonnée à une aide humanitaire massive alors que des initiatives individuelles émergeaient en son sein. Les européens impuissants et les Etats-Unis ne voulant pas intervenir, l'ONU fut un recours à la fois facile et inadapté. Ce n'est pas l'échec de l'organisation qui est à

(11) Le 31 mars 1995, trois résolutions modifient la FORPRONU. La résolution 981 crée l'ONURC (Opération des Nations Unies pour le Rétablissement de la Confiance en Croatie) qui remplace, pour la Croatie, la FORPRONU avec un mandat jusqu'au 30 novembre 1995. La résolution 982 proroge le mandat de la FORPRONU en Bosnie jusqu'au 30 novembre 1995. La résolution 983 transforme la FORPRONU en FORDEPRENU (Force de Déploiement Préventif des Nations Unies) en Macédoine.

(12) Cette force s'illustrera notamment par une riposte massive au bombardement par les serbes de Bosnie de Sarajevo le 28 août 1995. Néanmoins, elle ne parviendra pas à éviter la chute de Srebrenica le 11 juillet 1995 aux mains des serbes de Bosnie, cette "victoire" étant suivie d'une épuration massive de la population.

(13) Selon un bilan approximatif établi par Le Courrier International ( $n^{\circ} 280$ du 14 au 20 mars 1996) : 250000 morts, 3,5 millions de personnes déplacées à l'intérieur de l'ex-Yougoslavie (auxquelles il faut ajouter un million de personnes ayant trouvé refuge à l'étranger), 500000 personnes incarcérées, 50000 personnes torturées, 20000 femmes violées, 150 fosses communes découvertes. Il est nécessaire de préciser que 3 millions de mines antipersonnelles se trouvent en Bosnie, le bilan pourrait donc s'aggraver. 
souligner, c'est l'échec des principaux Etats membres de celle-ci. Comme ceci fut fréquemment souligné, une force de paix était présente pendant la guerre, une force de guerre sera présente pour assurer la paix (14). Sans doute a-t-on réussi à canaliser le conflit dans les limites de l'ex-Yougoslavie mais au prix d'un huis-clos où le droit des minorités est devenu inexistant face à une épuration ethnique qui n'était pas la conséquence de la guerre mais sa cause (15). S'agissait-il d'un conflit "insoluble» car à la fois atypique et multiforme? Beaucoup n'étaient pas loin de le penser, à commencer par les représentants des grandes puissances (16). La France, à l'inverse, fut souvent à l'origine d'initiatives (autorisation du recours à la force par l'OTAN en février 1994, création de la FRR...) dont la signature du traité de paix au Palais de l'Elysée est le symbole.

En désespoir de cause, tous les plans de paix se sont fondés sur deux principes : l'acceptation des faits accomplis au niveau des conquêtes territoriales et du nettoyage ethnique, et la partition de la Bosnie sur des critères ethniques (17). Le plan de paix négocié à Dayton ne dérogera pas à ces principes très simples mais la complexité du conflit va se répercuter sur la formulation de la paix : à conflit complexe, accords complexes. Ce que le Conseil de sécurité a résumé en constatant "le caractère unique, extraordinaire et complexe de la situation actuelle en Bosnie-Herzégovine, qui demande une réponse exceptionnelle" dans sa résolution 1031 du 15 décembre 1995 (paragraphe 39).

L'accord de paix signé à Paris le 14 décembre 1995 est en lui-même un accord complexe auquel il convient de joindre d'autres accords qui le précèdent ou le complètent. L'accord de Paris est un traité de près de 150 pages complété par 102 cartes qui se décompose en un accord cadre général assez bref suivi de 11 annexes qui constituent l'essentiel des dispositions applicables. A cet ensemble s'ajoutent des lettres d'accompagnement et des lettres annexes (à la suite de l'annexe $1 \mathrm{~A}$ sur les aspects militaires du règlement de paix et à la suite des 11 annexes), un accord sur l'apposition des paraphes et des signatures de l'accord-cadre et une déclaration finale commune(18)

(14) Le Secrétaire général des Nations Unies résume cette situation: "...ces dernières années, nous avons envoyé nos casques bleus sur plusieurs théâtres d'opération (...) avec pour mission de maintenir une paix qui n'existait pas."; dans : "L'ONU et les nouveaux conflits internationaux", Relations Internationales et Stratégiques, $\mathrm{n}^{\circ} 20$, hiver $1995, \mathrm{p}$. 19. B. Boutros GHaLl a également donné son point de vue sur le conflit yougoslave, en insistant notamment sur le plus long pont aérien de l'histoire à Sarajevo, dans Le Monde du 9 février 1996 ( La grandeur intacte de l'ONU »).

(15) Dès l'été 1992, le rapporteur spécial de l'ONU, T. MAZOWIECKI, indiquait : "La purification ethnique n'est pas le résultat des actions armées, elle est le but de cette guerre".

(16) L. Freedman estime que "la perversité de tels conflits ne semble avoir d'égale que leur insolubilité", mais qu'en tout état de cause, "il est toujours dangereux de se mêler de conflits locaux en prenant des demi-mesures"; dans : "Bosnie : le soutien à la paix a-t-il un sens?", Revue de l'OTAN, $\mathrm{n}^{\circ} 6$, novembre 1995, p. 19 et p. 22. Sur l'attitude des grandes puissances durant le conflit, on consultera le dossier de Relations Internationales et Stratégiques $\mathrm{n}^{\circ} 19$, automne 1995, pp. 69 à 138.

(17) Voir à ce propos:S. Dizdarevic : «Bosnie : la paix sans la démocratie", dans Manière de voir $\mathrm{n}^{\circ} 29$ ("Conflits fin de siécle"), Le Monde diplomatique, février 1996, pp. 48-50.

(18) Les annexes concernent les aspects militaires du règlement de paix (1A), la stabilisation de la région (1B), la démarcation entre les entités (2), les élections (3), la constitution (4), l'arbitrage (5), les droits de l'homme (6), les réfugiés et personnes déplacées (7), lá commission pour la conservation des monuments nationaux (8), les entreprises publiques de Bosnie-Herzégovine (9), la mise en œuvre civile (de l'accord) (10) et la force de police internationale (11). En outre, la Déclaration finale commune mentionne la question des pilotes français prisonniers des serbes de Bosnie dont la libération était posée comme condition par la France à la signature définitive de l'accord de paix. On se souvient que ceux-ci furent libérés deux jours avant la signature de l'accord. Le texte de l'accord (ainsi que d'autres documents mentionnés ci-après) se trouve dans le numéro hors série des Documents d'actualité internationale de février 1996 sur Les accords de paix concernant l'ex-Yougoslavie. 
(tous ces textes sont en vigueur dès leur signature). Les signataires de cet accord sont le Président de la République de Bosnie-Herzégovine (A. Izetbegovic), le Président de la République de Croatie (F. Tudjman) et le Président de la République Fédérative de Yougoslavie (Serbie et Monténégro) (S. Milosevic), ce dernier représentant également les serbes de Bosnie. Les membres du Groupe de Contact sont signataires en tant que témoin (19).

Ce traité est un accord à tiroirs dont l'accord-cadre général est le reflet et le résumć. Dans ce dernier, les trois Républiques affirment leur adhésion aux principes de base agréés lors de différents accords préparatoires et prennent acte de l'accord du 28 août 1995 autorisant la délégation de signature à la République Fédérative de Yougoslavie (RFY) au nom de la République Srpska (c'est-à-dire la république autoproclamée des serbes de Bosnie) avec obligation de mise en ouvre de l'accord. Après un rappel du respect des principes de la Charte des Nations Unies, de l'acte final d'Helsinki et des autres textes de l'OSCE, l'accord-cadre énumère les différentes annexes que les signataires s'engagent à respecter à l'aide d'une formule type. L'article X de l'accord cadre précise que la RFY et la République de Bosnie-Herzégovine se reconnaissent mutuellement mais que les aspects relatifs à leur reconnaissance mutuelle feront l'objet de décisions ultérieurs (des lettres identiques et croisées entre la RFY et la République de Bosnie concernant leur reconnaissance mutuelle seront jointes à l'accord de paix). La reconnaissance interviendra formellement le 14 décembre après la signature du traité. La Slovénie avait reconnu la RFY dès le 30 novembre alors que la Grèce et la Bosnie établissaient des relations diplomatiques le même jour.

L'accord général de paix ne peut se comprendre qu'avec l'éclairage des phases qui ont précédé ou qui vont suivre. En effet, il s'agit d'un ensemble très dense de "pré-accords" et de conférences qui illustrent toute la complexité du règlement de paix en Bosnie.

Des accords partiels ont mené aux négociations de Dayton. L'accord de Genève du 8 septembre 1995 pose le principe de la repartition du territoire (49\% pour les serbes et $51 \%$ pour la fédération croato-bosniaque). L'accord de New York du 26 septembre suivant sur les "principes institutionnels" complète celui du 8 septembre et précise l'organisation constitutionnelle de la Bosnie ainsi que les élections à venir. Le cessez-le-feu est contenu dans un accord du 5 octobre (entré en vigueur le 12 octobre) et sa durée originelle était de 60 jours (il s'agit du $36^{\mathrm{e}}$ cessez-le-feu depuis le début du conflit). Sur cette base, les négociations de Dayton vont pouvoir s'engager et vont donner lieu à une série d'accords intermédiaires et complémentaires à l'accord général de paix.

Le 10 novembre intervient un accord sur le renforcement de la fédération croato-musulmane entre le Président de la Bosnie et celui de la Fédération (K. Zubak) accompagnés par le Président de la Croatie comme témoin. Un accord sur le statut de la Slavonie orientale intervient le 12 novembre (Erdut et Zagreb) entre la Croatie et les sécessionnistes serbes, ce qui était impératif le mandat de l'ONURC s'achevant le 30 novembre.

A ces accords préliminaires viennent s'ajouter plusieurs conférences prévues par l'accord de paix selon les questions à traiter : la conférence de Londres ouverte les 8 et 9 décembre se charge des aspects civils du plan de

(19) F. Gonzalez pour la Présidence de l'Union européenne, J. Chirac pour la France, H. Kohl pour l'Allemagne, V. Tchernomyrdine pour la Russie, J. Major pour le Royaume Uni et B. Clinton pour les Etats-Unis. 
paix, la conférence de Bonn ouverte les 18 et 19 décembre entame une série de négociations sous l'égide de l'OSCE pour le désarmement et les mesures de confiance en ex-Yougoslavie, et la conférence de Bruxelles ouverte les 20 et 21 décembre se charge des aspects financiers de la reconstruction.

Le Conseil de sécurité des Nations Unies va jouer un rôle de légitimation de ces accords en adoptant plusieurs résolutions sur la base de ceux-ci. Il faut notamment mentionner les résolutions du 22 novembre qui suspendent l'embargo imposé à la RFY ainsi que l'embargo sur les armes (graducllement) en vigueur depuis septembre 1991(20) et l'importante résolution $1031 \mathrm{du}$ 15 décembre 1995 qui avalise la création d'une force multinationale de mise en œuvre de la paix (IFOR).

L'accord de paix est non seulement le résultat d'un ensemble de textes hybrides mais il est également d'une nature particulière. Il s'agit d'un accord "pratique» qui tente de régler empiriquement les nombreuses questions découlant du conflit mais qui ne pose aucunement le problème des responsabilités des acteurs de cette tragédie.

On a provoqué et attendu la coïncidence entre la situation sur le terrain et le découpage territorial qui avait déjà été proposé (plan Vance-Owen de 1993 ou celui du groupe de Contact en 1994) pour faire aboutir les négociations menant au plan de paix. Il fallait faire du cessez-le-feu une paix et de la paix un règlement durable. Dans ce sens, l'accord est «cynique " et ne pose pas comme véritable objectif une Bosnie pluriculturelle et tolérante malgré les affirmations de principe qui englobent le traité. Il se contente d'absorber toutes les contradictions existantes sous une apparence de logiques qu'il sera difficile de concilier. Sans doute ce malaise est-il accentué par l'objet du traité : s'agit-il de mettre fin à une guerre civile ou à une guerre internationale? En effet, si l'aspect international est présent depuis l'affirmation de la dissolution de la Yougoslavie et la naissance d'Etats en résultant, l'aspect interne n'est pas absent puisqu'il s'agit de séparer à l'intérieur d'un même Etat des communautés tentées (c'est un euphémisme) par un rattachement externe que d'autres Etats ont provoqué (21).

Très clairement, l'accord de paix est un traité évolutif soumis à la variabilité des situations en découlant(22). Aláin Joxe résume cette situation : "Il faut interpréter l'accord comme un texte 'empirique' appartenant à la culture du droit coutumier anglo-saxon, c'est-à-dire pouvant évoluer par la pratique»(23).

Face à cette nature particulière, à cet objet hybride, à ce caractère résolument évolutif, la modestie s'impose pour envisager successivement les aspects militaires et territoriaux du règlement de paix (I), les aspects civils (II) et l'objectif de la consolidation de la paix par le droit (III).

(20) Résolutions 1022 et 1023. La première sur la suspension des mesures économiques (elles seront levées ultérieurement) fut votée à l'unanimité, alors que la deuxième sur la levée de l'embargo sur les armes a entraîné l'abstention de la Russie.

(21) Selon P. HASSNER : "Bien des conflits sont des guerres civiles pour l'une des parties et des guerres interétatiques pour l'autre, et (...) elles changent de catégories selon le résultat de l'affrontement et selon le verdict de la communauté internationale", dans La violence et la paix (de la bombe atomique au nettoyage ethnique), éd. Esprit, Paris, 1995, p. 16.

(22) Les déclarations à l'issue de la signature du Traité de Paris le 14 décembre ne laissent aucun doute sur sa nécessaire évolution. Selon A. Izetbegovic : "La bataille va se poursuivre par la force des idées" (celui-ci estimant que le traité est une "médecine amère "), alors que J. Chirac avouait : "la paix reste à construire". 1995.

(23) "Dayton, cessez le feu prolongé ou paix sans principe?", Le Monde du 14 décembre 


\section{I \\ LA FIN DE LA GUERRE : LES ASPECTS MILITAIRES ET TERRITORIAUX DU REGLEMENT DE PAIX}

L'accord de paix tente, en premier lieu, de mettre fin au conflit qui sévit sur le territoire de la Bosnie. Les aspects militaires immédiats sont les premiers évoqués à la suite de l'accord-cadre général dans l'annexe 1A. Néanmoins, la perspective de faire taire les armes ne doit pas occulter la nécessaire construction d'une stabilité régionale à moyen et long terme. Cette volonté s'accomplit dans un cadre territorial où les nouvelles démarcations entre les entités à l'intérieur de la Bosnie s'apparentent à de véritables «frontières".

\section{A. Les aspects militaires immédiats : la vision sécuritaire}

L'opération mise en place pour garantir militairement le respect de l'accord de paix est, à bien des égards, une opération originale qui combine des innovations et qui se nourrit des expériences - et des erreurs - des opérations précédentes. La création, le statut, les missions et l'échéancier prévus par l'annexe $1 \mathrm{~A}$ de l'accord de paix montrent toutes les difficultés, les incertitudes et les nouveautés de cette opération.

L'opération créée en Bosnie pour "une durée d'environ une année" (article $1^{\text {er }}$ ) doit succéder à la FORPRONU quand le Conseil de sécurité aura décidé de la création, par une résolution, d'une force multinationale chargée de la mise en œuvre de l'accord. Cette force dénommée IFOR (Implementation Force) doit provenir des pays membres de l'OTAN ainsi que d'autres Etats en accord avec l'OTAN, mais sera sous la direction et le contrôle du Conseil de l'Atlantique Nord (CAN) "via la chaîne de commandement de l'OTAN" (article $1^{\mathrm{er}}$, alinéa b). Plus précisément, l'article VI indique que "le Conseil de sécurité des Nations Unies est invité à autoriser tous les Etats membres ou les organisations régionales à créer l'IFOR, agissant en vertu des dispositions du chapitre VII de la Charte des Nations Unies".

Cette opération sera, en réalité, préparée des le mois de septembre (24) et comportera de nombreux détours par les autorités nationales et par les instances internes de l'OTAN pour se concrétiser. Mais la légitimation de l'opération doit provenir de l'ONU qui sert, en l'espèce, de cadre général international pour permettre à l'opération de se réaliser sans pour autant que son rôle déborde le cadre du transfert de la FORPRONU à l'IFOR dans le domaine militaire.

C'est la résolution 1031 du Conseil de sécurité du 15 décembre 1995, adoptée à l'unanimité, qui crée la force multinationale de mise en œuvre de la paix. "Agissant en vertu du chapitre VII de la Charte", selon la formule désormais consacrée, le Conseil de sécurité autorise les Etats membres agissant par l'intermédiaire de l'organisation visée à l'Annexe $1 \mathrm{~A}$ ou en coopération avec elle à créer "une force multinationale de mise en œuvre de la paix". L'habilitation donnée par le Conseil de sécurité est large puisqu'elle «autorise les Etats membres à prendre, à la demande de l'IFOR, toutes les mesures nécessaires pour défendre la Force ou pour l'aider à

(24) Il s'agit du plan 40103 de force d'interposition préparé par l'OTAN, voir la chronique des faits internationaux, RGDIP 1995/4, p. 1010. 
accomplir sa mission et reconnaît le droit qu'a la Force de prendre toutes les mesures nécessaires à sa défense en cas d'attaque ou de menace" (II, paragraphe 17). En outre, la résolution indique qu'à la date du transfert entre l'IFOR et la FORPRONU, toutes les résolutions antérieures cesseront de produire leurs effets et que les Etats peuvent utiliser "tous les moyens nécessaires" (encore une formule consacrée) pour aider au retrait de la FORPRONU. Selon un scénario sans faille, le 15 décembre sont également publiées "Les conclusions et la Déclaration du Conseil européen sur l'exYougoslavie» qui avalisent l'ensemble du processus de paix signé la veille (25).

La résolution 1031 marque la fin du mandat de la FORPRONU qui, par prudence, avait été reconduit jusqu'au 31 janvier 1996 par la résolution 1026 du 30 novembre 1995. Le transfert s'effectuera le 19 décembre, soit dans les 96 heures suivant la résolution 1031 comme ceci avait été prévu. Prenant acte de la fin "officielle" du conflit, le gouvernement de Sarajevo publiera le 22 décembre un décret mettant fin à l'état de guerre en vigueur depuis juin 1992. Néanmoins, celui-ci sera remplacé par un "état de danger de guerre".

L'IFOR est une impressionnante force de plus de 60000 hommes composée de trois divisions multinationales avec un Etat "chef de file" et une zone géographique propre pour chacune de ces divisions (26). Le commandement unique de cette force est assurée, sur le terrain, par l'Amiral américain L.Smith, commandant des forces de l'OTAN pour le sud de l'Europe, secondé par le général français Janvier (ancien commandant de la FORPRONU). L'ensemble est commandé depuis Mons, en Belgique, par le général américain G. Joulwan. Néanmoins, si les contingents sont sous "contrôle opérationnel de l'OTAN", ils restent sous commandement national. Dès le 27 novembre, les chefs d'état major réunis à Bruxelles avaient approuvé la synthèse faite par le Comité militaire de l'OTAN et prévoyant les détails de l'organisation de l'OTAN pour cette mission d'une ampleur exceptionnelle (27).

Les missions conférées à l'IFOR sont précisées dans l'annexe 1A. Si elles sont axées autour de quelques points forts, elles comportent de multiples possibilités et quelques points d'ombre (dont la question du soutien au Tribunal Pénal International, cf. infra). On y retrouve un souci sécuritaire immédiat qui donne l'impression de cantonner l'IFOR à quelques missions précises dont l'ampleur ne peut s'inscrire dans la durée.

On peut résumer les différentes missions en estimant qu'elles sont au nombre de trois : contrôler la cessation des hostilités, vérifier le retrait des

(25) Texte dans $D A I$ hors série de février 1996, op. cit., pp. 66-67.

(26) Les effectifs (approximatifs) sont les suivants: Etats-Unis (20000), Royaume Uni (12 000), Francc (10 000), Russie (4 000), Allemagne (5 000), Italie (2 500), Espagne (1 500) ainsi que la participation du Portugal, de l'Autriche, du Canada, de la Finlande, de la Suède, de la Norvège, de la Hongrie, des Etats baltes, de la République Tchèque, de la Pologne, de la Roumanie, de la Slovaquie, de l'Ukraine, du Pakistan, du Bangladesh, de la Jordanie, de la Malaisie, du Maroc et de l'Egypte. La zone Nord (PC à Tuzla) est sous la responsabilité des Etats-Unis, la zone Sud-Ouest (PC à Gornji Vakuf) sous la responsabilité du Royaume Uni et la zone Sud-Est (PC à Sarajevo et Mostar) sous la responsabilité de la France. Le financement de cette Force se fait selon un barème institué par l'OTAN : la France contribuerait pour environ $16 \%$ du total, ce qui représenterait 4 milliards de francs pour 1996.

(27) La France reprendra sa place au Comité militaire de l'OTAN le 5 décembre 1995. Il s'agit de la plus haute instance militaire de l'OTAN placée sous l'autorité politique du Conseil de l'Atlantique Nord (CAN) et du Comité des plans de défense (CPD). La France en était absente depuis son départ des organes intégrés de l'OTAN en 1966. 
forces étrangères et s'assurer du redéploiement effectif des forces en présence au-delà des limites prescrites par l'accord de paix.

La cessation des hostilités résulte de l'accord du 5 octobre 1995 et proscrit toute action offensive définie comme "une action incluant l'envoi de forces ou la pratique de tirs au-delà des lignes de chaque partie" (article II, alinéa 1). La situation sur le terrain étant relativement obscure avec la présence d'éléments armés ayant des statuts différents, l'annexe 1A précise que la cessation des hostilités concerne l'ensemble du personnel et des organisations militaires incluant les groupes civils armés, les éléments de la garde nationale, toutes les forces militaires de réserve, tous les éléments de police militaire ainsi que la police spéciale du ministère de l'intérieur de Bosnie (MUP). Ne sont pas concernés : la FORPRONU, l'IFOR et la Force de police internationale (constituée en vertu de l'annexe 11). Il s'agit finalement de désarmer tout groupe civil armé et ceci dans un délai de trente jours. De la même manière, l'article III impose le retrait des forces étrangères dans un délai de trente jours à compter de l'entrée en vigueur de l'accord. Ceci vise bien sûr les forces régulières de la RFY et de la République de Croatie mais aussi des "combattants " venus d'autres Etats et notamment des pays islamistes comme l'Iran qui souhaitaient faire de la Bosnie un nouveau terrain de guerre sainte. Enfin le redéploiement des forces au-delà de la ligne de cessez-le-feu derrière une zone de séparation est prévu en trois phases selon un échéancier minutieux qui constitue une des parties les plus précises de l'annexe 1A. C'est l'IFOR qui doit se charger du marquage de la ligne de cessez-le-feu et de sa zone de séparation, ainsi que de la ligne de démarcation entre les entités et sa zone de séparation (article VI, alinća 2). En effet, la ligne de cessez-le-feu ne correspond pas toujours à la ligne de démarcation prévue et c'est notamment le cas pour Sarajevo dont les quartiers occupés par les serbes de Bosnie devront être évacués.

Ces trois missions essentielles sont complétées par des missions annexes dont certaines ne sont pas sans ambiguïté. Ainsi, l'article IX demande à l'IFOR, en collaboration avec le Comité International de la Croix-Rouge (CICR) de veiller à la libération et au transfert des prisonniers dans les trente jours après le transfert d'autorité entre la FORPRONU et l'IFOR (des listes exhaustives devant être établies dans les 21 jours). Le cas des prisonniers est ainsi traité séparément de celui des réfugiés et personnes déplacées (annexe 7) et rentre directement dans le volet militaire de l'accord (28). Des prisonniers furent libérés les 27 et 28 janvier 1996 (environ 500 personnes) moins quelques prisonniers soupçonnés de crimes de guerre. Cette rétention donna lieu à des incidents diplomatiques entre les entités. Un accord complémentaire entre la Fédération de Bosnie-Herzégovine et la République Srpska du 26 janvier 1995 permit de régler des aspects spécifiques concernant des prisonniers entre ces entités.

Mais l'article VI apporte également un éclairage intéressant sur les missions annexes de l'IFOR en indiquant que la Force "a le droit de remplir des tâches de soutien" dans la limite de sa tâche principale et de ses ressources : aider à l'organisation d'élections justes et démocratiques, faciliter le mouvement des organisations humanitaires, aider le HCR et les autres

(28) La libération de tous les non combattants détenus est envisagée dans des lettres annexées à l'accord de paix. Il y est prévu la libération de ces personnes conformément à la résolution 1019 du Conseil de sécurité du 9 novembre 1995 ainsi que la fermeture de tous les camps de détention avec un accès du CICR. 
institutions internationales, éviter les heurts entre la population et les réfugiés ou encore, contrôler le déminage. Il est clair que l'OTAN n'aura pas de missions de police civile ni humanitaire et l'article VI reste muet sur l'aide à apporter au Tribunal Pénal International (TPI), ce qui donnera lieu à quelques "flottements" dans l'application de l'accord de paix.

L'ensemble des missions de l'IFOR présentant incontestablement des risques importants, l'annexe $1 \mathrm{~A}$ prévoit la création d'une Commission Militaire Mixte (CMM) (article VIII) qui doit étudier tous les cas de violation de l'accord, le non respect du cessez-le-feu et aider le Commandant de l'IFOR dans les décisions à prendre (29). De la même manière, le Supplément $\mathrm{B}$ à l'annexe 1A est un accord entre la République de Bosnie et l'OTAN concernant le statut de la Force et de son personnel. Outre les privilèges et immunités classiques en référence à la convention des Nations Unies du 13 février 1946, l'accord s'attache à être très précis sur la définition des termes («OTAN», «personnel de l'OTAN»...) et sur les juridictions compétentes en cas d'infraction de la part de la Force(30). Il est vrai que les missions ne sont pas sans risques (31) malgré un échéancier minutieux qui constitue un des points les plus remarquables de l'accord de paix.

La mission de redéploiement des forces (article IV) est d'une grande précision. Trois phases sont minutieusement prévues avec un échéancier strict. Dans un délai de trente jours à compter du transfert d'autorité, les forces de part et d'autre de la ligne de cessez-le-feu doivent se retirer derrière une zone de séparation (précisée dans la carte du supplément A à l'annexe 1A(32)) de deux kilomètres. Il existera ainsi une zone de sécurité de quatre kilomètres de large (cela représente environ 1000 kilomètres de ligne de démarcation à surveiller). Durant cette première phase, des dispositions spécifiques s'appliquent à Sarajevo et à Goradze avec notamment le problème de la construction d'une route reliant le couloir de Goradze à Sarajevo. Le 19 janvier, cette première échéance était respectée même si la construction de cette route pose de multiples problèmes. La deuxième phase doit s'effectuer dans un délai de $\mathbf{4 5}$ jours à compter du transfert d'autorité et concerne la question des zones transférées à une autre entité pour des lieux spécifiés où la ligne de démarcation ne suit pas la ligne de cessez-le-feu agréée (cet aspect concerne surtout Sarajevo). L'accord est très détaillé sur les distances entre les zones et les délais à respecter. Le 3 février 1996, cette phase était

(29) La composition de la CMM reflète le difficile équilibre à instaurer entre les parties. Elle comprend notamment le commandant militaire en chef des forces de chaque partie en Bosnie, ceux-ci pouvant se faire aider par des civils comme conseil... Néanmoins, cette CMM est simplement considérée comme un organe consultatif, le commandant de l'IFOR gardant toute latitude dans ses décisions.

(30) Ainsi, l'alinéa 7 nous indique que le personnel militaire de l'OTAN sera "en toutes circonstances et à tout moment, soumis à la juridiction exclusive de leurs élements nationaux respectifs, au regard de tous crimes, délits ou infractions éventuellement commis par eux sur le territoire de la République de Bosnie-Herzégovine...". Sans doute le douloureux souvenir d'un statut insuffisamment précisé (notamment en Somalie pour les forces de l'ONU) a-t-il influé sur la rédaction de cet accord.

(31) On peut citer notamment la surveillance (dans le secteur américain) du couloir de Posavina (avec les agglomérations de Brcko, Orasje et Gradacac) revendiqué par toutes les entités et dont le sort est lié à un arbitrage prévu dans l'accord de paix; le transfert des villes croates de Sijovo et Mrkonjic Grad à la partie serbe (dans le secteur du Royaume Uni) ou la situation de la ville de Sarajevo, l'ouverture de la route de Sarajevo à Goradze, la surveillance du corridor de Prevlaska (accès serbe à la mer adriatique) ou la délicate situation de la ville de Mostar divisée entre les croates et les musulmans (dans le secteur sous surveillance de la France).

(32) Voir la carte annexée au numéro hors série des DAI, op. cit. 
également respectée. Enfin la troisième phase couvre une période de $\mathbf{1 2 0}$ jours à compter du transfert d'autorité et concerne le retrait de toutes les armes lourdes (celles-ci sont précisées dans l'accord) et de toutes les forces vers des zones de cantonnement et de stationnement ainsi que la démobilisation des forces. L'IFOR peut utiliser la force pour imposer ce retrait.

A cet échéancier vient s'ajouter celui du transfert complet de souveraineté de plusieurs zones de Bosnie entre la République Srpska et la fédération croato-musulmane dont la réunification de Sarajevo le 19 mars est le symbole avec l'abandon par les serbes de Bosnie du quartier de Grbavica.

$\mathrm{Si}$ le calendrier fut globalement respecté, certains points durent néanmoins être précisés lors de la réunion, à Rome, des signataires de l'accord, les 17 et 18 mars 1996. Outre la question des criminels de guerre, l'échec de la réunification de la ville de Mostar et la présence de moudjahidines iraniens en Bosnie furent les deux principaux points abordés. L'accord résultant de ce sommet semble avoir aplani tous les points de désaccord, les différentes parties s'engageant à respecter la lettre de l'accord de paix.

Il est difficile de dresser un bilan des missions de l'IFOR alors que celles-ci ne sont pas achevées mais quelques enseignements peuvent être d'ores et déjà dégagés.

Tout d'abord, l'IFOR constitue un ensemble d'innovations dans le domaine des opérations qui ont pour objet d'imposer et de garantir la paix. L'opération "Effort concerté" (Joint Endeavour) est la première opération terrestre de l'OTAN depuis 1949 dans un cadre autre que celui qui était envisagé lors de la création de l'OTAN. Pour la première fois, elle sort de sa zone d'intervention. Pour la première fois, elle va travailler avec des Etats qui étaient ses ennemis potentiels avant la fin de la guerre froide (dont la Russie est le symbole), avec des Etats européens non membres de l'Alliance Atlantique (y compris des pays neutres comme l'Autriche ou la Suède) et avec des pays "hors champs" comme le Maroc, le Bangladesh ou la Malaisie. De plus, cette opération lui permet d'expérimenter de nouvelles techniques internes à l'organisation (33) et surtout le concept de "maintien de la paix multifonctionnel» qui résulte directement d'une adaptation à l'OTAN des expériences et des propositions de l'ONU en matière de maintien de la paix. S'inspirant des propositions de l'Agenda pour la paix de 1992 du Secrétaire général des Nations Unies qui distinguait plusieurs phases dans le maintien de la paix (diplomatie préventive, rétablissement de la paix, maintien de la paix, consolidation de la paix) et de l'apparition plus récente d'un concept d'imposition de la paix comportant une forte connotation coercitive (désarmement et sanctions), l'OTAN tente de dégager une notion de paix multifonctionnelle incluant une variété d'opérations dont l'IFOR serait une représentation (34). En effet, ce concept doit réunir deux caractéristiques qui

(33) Comme le "Grand quartier général des puissances alliées en Europe" (SHAPE) dont le but est de traduire les instructions des autorités politiques de l'OTAN en directives opérationnelles claires et précises pour les commandements subordonnés, voir Général JouLWAN : "Le SHAPE et l'IFOR : s'adapter aux besoins de demain ", Revue de l'OTAN, vol. 44, n 2, mars 1996, pp. 6 à 9 .

(34) Ces opérations inclueraient des fonctions militaires traditionnelles (séparation des forces, observation...), des aspects politiques en matière de négociation, des aspects touchant les droits de l'homme, l'assistance humanitaire, le reconstruction... Un "Groupe ad hoc sur la coopération en matière de maintien de la paix" existe au sein de l'OTAN depuis 1993. Celui-ci réunit les membres de l'OTAN, les membres de l'ex-Pacte de Varsovie, divers Etats ainsi que l'OSCE et l'ONU. Voir : D. LIGHTBURN : "L'OTAN et le défi du maintien de la paix multifonctionnel ", ibid., pp. 10 à 14. 
existent pour la Bosnie : des conditions de sécurité complexes, imprévisibles et dangereuses, et une situation humanitaire grave (famine, génocide, réfugiés...).

Ces innovations expliquent les difficultés rencontrées durant les négociations pour les faire coexister au sein d'une même opération. La France a fait un pas en acceptant de reprendre sa place au sein du Comité militaire. Le 6 décembre, les députés allemands du Bundestag ont approuvé le déploiement de 4000 soldats en ex-Yougoslavie, ce qui représente la plus importante mission à l'étranger depuis la deuxième guerre mondiale même si les troupes seront «non combattantes " et stationnées à Split en Croatie pour des raisons historiques. A la suite d'un vote du Parlement le 5 décembre 1995, la Hongrie a envoyé 500 soldats "non combattants" en Croatie et le territoire de l'Etat sert de base arrière au contingent américain de l'IFOR. Enfin, il convient de mentionner la participation de la Russie sous une forme un peu particulière puisque qu'un Comité consultatif réunissant les membres de l'Alliance et la Russie fut constitué pour l'occasion, les troupes russes n'étant pas officiellement intégrées à l'OTAN mais répondant aux ordres du commandement militaire américain. Ce compromis s'imposait à la suite du succès mitigé du Partenariat pour la paix voulu par l'OTAN et de la volonté de la Russie d'imposer pour cette opération la "double clef" (OTAN-ONU) dont les Etats-Unis ne voulaient pas.

Le rôle central des Etats-Unis est tout à fait remarquable dans la constitution de l'IFOR et explique les conditions imposées à l'opération. C'est à la suite d'une allocution le 27 novembre 1995 que le Président Clinton indique l'intérêt pour les Etats-Unis de participer à une telle mission et qu'il précise le "profil" de l'opération. Il s'agit, pour les soldats américains d'une "mission claire, limitée et réalisable" impliquant des directives claires concernant notamment les ordres d'engagement dans les combats (les soldats sont autorisés à tirer en premier si leur situation est menacée). Ces conditions étaient nécessaires pour obtenir l'aval du Sénat américain. Le 13 décembre, celui-ci approuva (par 69 voix contre 30 ) l'envoi d'un contingent en Bosnie à condition que les Etats-Unis ne s'engagent pas dans des activités non militaires (reconstruction du pays, réinstallation des réfugiés...) et qu'ils s'engagent à former une armée bosniaque pour équilibrer les forces en présence après le départ de l'IFOR (35).

Les Etats-Unis privilégient, par conséquent, une vision sécuritaire immédiate et un engagement limité dans le temps et dans les missions. Or, cette vision ne peut suffire même si elle s'avère indispensable dans un premier temps: "L'action militaire ne peut jamais suffire; au mieux, elle peut créer les conditions nécessaires à une issue politique plus favorable. Telle est l'essence d'une mission de stabilisation»(36). C'est la raison pour laquelle la vision politique à plus long terme privilégiée par les européens trouve également un écho dans l'accord de paix, même si l'on n'y retrouve pas la précision et l'engagement qui caractérisent les aspects militaires immédiats.

(35) En échange de l'aval du Congrès, le Président Clinton a accepté le projet de budget qu'il désapprouvait auparavant.

(36) L. Freedman: "Bosnie : le soutien à la paix a-t-il un sens? ", Revue de l'OTAN, op. cit., p. 22. 


\section{B. La perspective d'une stabilité régionale : la vision politique}

Les Etats-Unis envisagent l'équilibre des forces en présence pour garantir la paix. De cette façon, ils encouragent une logique de dissuasion qui est aussi une logique de partition de l'Etat bosniaque. A l'inverse, durant les négociations, les européens ont tenté de privilégier des mesures de confiance, de réconciliation en insistant sur les aspects civils. L'accord de paix laisse ouverte les deux options et dans un souci très diplomatique de ne heurter la sensibilité d'aucune des deux tendances, la stabilité régionale constitue l'annexe 1B.

L'accord sur la stabilisation de la région contenu dans l'annexe 1B concerne à la fois la RFY, la Croatie, la Bosnie-Herzégovine, la Fédération de Bosnie-herzégovine et la République Srpska. En l'espèce, les «entités» de la Bosnie sont signataires à part entière de l'accord au même titre que les trois Etats (la RFY étant, de plus, signataire au nom de la République Srpska). L'annexe 1B vise la stabilité régionale et le nécessaire contrôle des armements pour "parvenir à des niveaux de forces de défenses équilibrés et stables les plus bas possibles (...) avec la nécessité d'éviter une course aux armements dans la région» (article I). Cet objectif général est complété par une série de mesures de confiance et de sécurité en Bosnie (article II) ainsi que des mesures de contrôle des armements au niveau sous régional (article IV). Il y est prévu globalement que des négociations devront s'engager (dans un délai de sept jours à dater de l'entrée en vigueur de l'accord) entre la Bosnie et ses deux entités, sous l'égide de l'OSCE, pour parvenir à des mesures de confiance dans l'esprit du document de Vienne de 1994 sur les mesures de confiance et de sécurité de l'OSCE. L'objectif est de parvenir à un accord dans un délai de 45 jours sur un ensemble de mesures (37). Au-delà du cadre bosniaque, l'article III envisage un accord plus large sur la sécurité et les mesures de confiance dans la région limitant l'importation des armes et des armes lourdes dans des délais prescrits par l'accord (délai de 90 jours pour les armes et de 180 jours pour les armes lourdes). Ces délais pourront être raccourcis si un accord intervient avant selon le processus prévu à l'article IV. En effet, cet article prévoit une série d'accords sur les niveaux d'armements et sur les effectifs militaires dans les trente jours avec l'application automatique de plafonds d'armements dans les 180 jours si aucun accord n'est intervenu (38).

Au-delà d'une certaine complexité apparente, on peut distinguer la volonté de conclure des accords concernant la sécurité et les mesures de confiance en Bosnie, la sécurité et les mesures de confiance dans la région et des accords concernant le contrôle des armements à la fois au sein de la Bosnie et au niveau régional. L'ensemble du processus ayant pour cadre l'OSCE.

Le 8 décembre 1995 (à Budapest), l'OSCE prenait une «Décision pour l'action de l'OSCE, pour la paix, la démocratie et la stabilité en Bosnie-Herzégovine»(39) où le Conseil ministériel acceptait les demandes des négocia-

(37) L'article II cite notamment: les restrictions sur les manœuvres militaires dans certaines zones, les restrictions sur la localisation des armes lourdes, le cantonnement et le stationnement des forces et des armes lourdes, le démantèlement des groupes civils armés, la surveillance de la capacité de production d'armements, l'échange d'informations sur les cinq catégories d'armes définies dans le traité FCE (Forces Conventionnelles en Europe).

(38) Avec une proportion de $75 \%$ pour la RFY, $30 \%$ pour la Croatie et $30 \%$ pour la Bosnie (avec un rapport interne de deux pour la Fédération contre un pour la République Srpska).

(39) Texte dans le numéro hors série des $D A I$, op. cit., pp. 16-17. 
teurs de Dayton concernant l'OSCE. Elle créait à cet effet une mission en Bosnie pour une durée d'au moins un an. Le 13 décembre 1995, une "Déclaration sur le processus de stabilité et de bon voisinage en Europe du Sud-Est" (à Royaumont) envisageait une réflexion plus large dans l'esprit du Pacte de Stabilité adopté lors de la conférence des 20 et 21 mars 1995 et prévoyait de réunir une conférence sur la stabilité en Europe du Sud-Est(40). Mais c'est la conférence de Bonn qui se tiendra le 18 décembre 1995 (dans le délai de sept jours prescrit par l'accord) qui va ouvrir le véritable cycle des négociations sur les mesures de sécurité et le niveau d'armement. Cette conférence sous l'égide de l'OSCE va décider des objectifs et du calendrier des négociations pour l'application de l'accord de paix (particulièrement de l'article IV de l'annexe 1B)(41). Dans le respect des délais, la conférence suivante aura lieu à Vienne du 4 au 26 janvier 1996, un accord sur les mesures de confiance étant adopté le 26 janvier. Cet accord permet des inspections réciproques du niveau d'armement dans le cadre régional et ouvre la voie à un accord sur le désarmement. Ce dernier est rendu difficile par l'appréciation différente de la situation entre les Etats-Unis et l'Europe. Le plus bas niveau d'armement et de forces de défense possibles prévu dans l'accord se heurte à la volonté américaine - en dehors de l'accord de paix d'armer et de former l'armée bosniaque. Une conférence à Ankara (le 15 mars) a entériné le désaccord sur cet aspect du traité. Le processus en cours risque de connaître quelques soubresauts si les pays occidentaux ne parviennent pas à s'entendre.

L'accord de paix sur la Bosnie ne peut être isolé de conflits régionaux où les mêmes acteurs sont impliquées et qui rétroagissent sur la stabilité de ce fragile Ftat. C'est le cas du conflit en Slavonie orientale qui a donné lieu à un accord dit «fondamental» le 12 novembre 1995 entre les serbes de Slavonie et la Croatie. Cet accord place directement la Slavonie sous la tutelle de l'ONU avec la mise en place d'une administration transitoire et d'une force de maintien de la paix (42). La résolution 1025 du 30 novembre 1995 réaffirme que le territoire de la Slavonie fait partie intégrante de la République de Croatie et demande au Secrétaire général d'envisager le remplacement de l'ONURC par une opération transitoire telle qu'elle est prévue dans l'accord du 12 novembre. C'est la résolution 1037 du 15 janvier 1996 (date de la fin du mandat de l'ONURC) qui opère officiellement ce transfert. La résolution constate que la situation en Croatie «continue de constituer une menace à la paix et à la sécurité internationales " et "agissant en vertu du chapitre VII de la Charte...", elle décide de créer, pour une période initiale d'un an, une opération de maintien de la paix comportant des aspects militaires et civils sous le nom d'ATNUSO (Administration Transitoire des Nations Unies pour la Slavonie Orientale, la Baranja et le Srem occidental). Cette opération composée d'environ 5000 casques bleus remplira les missions indiquées dans l'accord du 12 novembre et pourra coopérer avec l'IFOR. Ce règlement du conflit en Slavonie ne peut être dissocié du règlement de paix en Bosnie car il est indispensable à la stabilité régionale et concerne également les serbes et les croates. Tout dérapage en Slavonie risquerait, par ricochet, d'avoir de graves conséquences sur le

(40) Ibid., p. 22

(41) Ibid., p. 67.

(42) L'accord prévoit notamment la démilitarisation de la région dans les 30 jours, le retour dans leur foyer des personnes déplacées avec la restitution des biens (ou l'indemnisation), la formation d'une police civile, l'organisation d'élections sous la surveillance de l'ONU et de l'OSCE. Voir le texte de l'accord dans le numéro hors série des DAI, op. cit., pp. 13-14. 
processus de paix en Bosnie. D'autant que la stabilité régionale dépend de la stabilité territoriale dont l'accord de paix fait un objectif majeur.

\section{Les nouvelles "frontières": la ligne de démarcation imposée par l'accord}

L'annexe 2 de l'accord de paix est consacré à la ligne de démarcation entre les entités (inter-entity boundary line) et concerne uniquement la République de Bosnie, la Fédération de Bosnie-Herzégovine et la République Srpska(43). La ligne de démarcation est indiquée sur une carte figurant dans le supplément à l'annexe (44). Ce supplément acquiert une valeur juridique certaine puisque l'article VII de l'annexe nous indique qu'il fait partie intégrante de l'accord. Tout réajustement de la ligne de démarcation devra se faire par accord mutuel entre les entités et le commandant de l'IFOR devra être obligatoirement consulté durant la période où la Force est en Bosnie (article II). La situation des fleuves ou des rivières changeant de lits est même envisagée (article III). Mais c'est l'article IV qui précise les modalités les plus importantes concernant la délimitation et le marquage. Il y est notamment précisé que ce sont les lignes et les zones définies par les cartes et les documents agréés par les Parties qui font foi et non l'emplacement physique des marquages. Une commission mixte est prévue pour préciser éventuellement la ligne de démarcation. Le transfert des pouvoirs en fonction de la ligne de démarcation agréée devra se réaliser dans les 45 jours suivant le transfert d'autorité entre la FORPRONU et I'IFOR (article VI).

La zone de Brcko fait l'objet d'un traitement à part dans l'article $\mathrm{V}$ de l'annexe 2. Cette portion litigieuse qui constitue un corridor reliant la partie occidentale à la partie orientale de la République serbe de Bosnie doit faire l'objet d'un "arbitrage contraignant" (article V, alinéa 1) accepté par les Parties. Six mois, au plus tard, après l'entrée en vigueur de l'accord, un arbitre sera désigné par la Fédération de Bosnie-Herzégovine, un par la République Srpska et un troisième d'un commun accord dans les trente jours (ce dernier sera le Président du Tribunal arbitral). A défaut, c'est le Président de la Cour internationale de Justice qui le désignera. Les règles d'arbitrage de la CNUCDI seront applicables sauf accord contraire et la décision sera rendue au plus tard un an après l'entrée en vigueur de l'accord. Celle-ci sera "définitive et obligatoire et les Parties devront la mettre en application sans délai " (article V, alinéa 3).

L'annexe 2 ne règle pas tous les aspects territoriaux, la ligne de démarcation consacrant essentiellement la ligne de cessez-le-feu comme ligne de partage. D'autres accords sont venus compléter les aspects territoriaux. C'est le cas de l'accord de mise en place de la Fédération de Bosnie-Herzégovine conclu à Dayton le 10 novembre 1995 entre le Président de la République de Bosnie (A. Izetbegovic) et le Président de la Fédération (K. Zubak) avec le Président de la Croatie comme témoin (ce dernier a seulement "approuvé»

(43) Néanmoins, les signataires sont la RFY, la Croatie et la République de Bosnie-Herzégovine, la Fédération de Bosnie-Herzégovine et la République Srpska apposant seulement un visa.

(44) Il s'agit d'une carte routière générale au $600000^{\mathrm{e}}$ et surtout d'une carte topographique au $50000^{\mathrm{e}}$ établie par les Etats-Unis, ce qui rend la ligne précise à 50 mètres près (selon l'article IV, alinéa 1). On pourra consulter l'excellente série de cartes jointes au dossier sur le conflit dans Manière de voir $\mathrm{n}^{\circ} 29$, op. cit., pp. 49 et $\mathrm{s}$.; ainsi que la série de cartes sur la répartition ethnique dans Le Monde du 23 novembre 1995. Nous reproduisons à la page suivante une carte générale montrant la partition de la Bosnie (Le Monde du 20 décembre 1995). 
Carte des zones militaires et de la délimitation entre les entités de Bosnie-Herzégovine à la suite de l'accord de paix du 14 décembre 1995 (Le Monde du 20 décembre 1995)

l'accord). Celui-ci a pour but de relancer sur des bases plus solides la Fédération crée le $1^{\text {er }}$ mars 1994 par les accords de Washington. Le préambule de l'accord du 10 novembre reconnaît le mauvais fonctionnement de la Fédération et demande au HVO (parti représentant la "Herzeg-Bosna" autoproclamée en août 1994 par les croates de l'ouest de la Bosnie et seulement reconnue par Zagreb) de transférer ses compétences à la Fédération. Lors des élections du 21 octobre 1995 en Croatie, les croates de la Bosnie ont pu voter au titre de la diaspora, ce qui augurait mal de la suite de la Fédération. Le nouvel accord s'efforce de répartir strictement les compétences entre les Parties (45) (mais une armée fédérale unifiée est prévue) et indique clairement que l'accord sera modifié pour être compatible

(45) La politique étrangère, le commerce extérieur, la politique douanière, la politique monétaire et commerciale... à la République de Bosnie; la défense, les affaires intérieures, la justice, l'énergie, les communications... à la Fédération. 
avec l'accord général de paix sur la Bosnie (46). Au-delà des grands principes posés, le statut de la ville de Mostar est l'un des enjeux essentiels de cet accord.

Mostar est le symbole de la division entre les croates et les musulmans de Bosnie. Sa réunification s'avère par conséquent indispensable pour donner une crédibilité à l'accord du 10 novembre. Des ministères, des séances du gouvernement de la Fédération (en alternance avec Sarajevo) y sont prévus. Mais surtout, il faut tenter de préserver l'unitć de la ville administrée par l'Union européenne (47) depuis le mémorandum du 6 juillet 1994 : des élections, une force de police unifiée... doivent y pourvoir. En février 1996, le projet prévoyant un centre ville multi-ethnique fut néanmoins violemment rejeté par les croates.

La transformation des quartiers serbes de Sarajevo en ville fantôme lors de la "réunification" de la capitale le 19 mars 1996 est une autre illustration de la coïncidence entre les divisions ethniques et territoriales que les accords proscrivent sans pouvoir les empêcher. Dès lors, on peut s'interroger sur la nature des limites territoriales à l'intérieur de la République de Bosnie : simple limite ou véritable frontière?

Une impression à peine diffuse se dégage du traité de paix : on prévoit les "limites" internes de la Bosnie comme de véritables frontières internationales avec toutes les garanties qui s'attachent à ce type de délimitation. De plus, ces «frontières" entérinent clairement un partage ethnique malgré les affirmations concernant le retour des réfugiés (cf. infra). La fiction est maintenue et les principes sont saufs : les frontières d'un Etat européen n'auront pas été modifiées par la force puisque l'intégrité de l'Etat bosniaque est proclamée en dépit des divisions internes. Pourtant, les lapsus trop nombreux réintroduisent la réalité dans cette fiction: A. Izetbegovic peu satisfait du statut du corridor (ce terme, en lui-même est déjà un mauvais souvenir) de Posavina parle de «frontières" avec la République serbe de Bosnie (48). L'artifice sémantique et institutionnel de la ligne de démarcation fait long feu.

Pourra-t-on, dès lors, empêcher une future partition de la Bosnie? Les garanties sur l'unité de l'Etat bosniaque paraissent insuffisantes pour éviter ce qui pourrait ressembler à un phénomène désormais bien connu en droit international : la sécession, voire la dissolution d'un Etat aux limites internes bien définies. En l'espèce, la configuration est un peu particulière puisqu'il s'agit d'un Etat unitaire incluant une Fédération et une République, mais il sera difficile d'affirmer un quelconque manquement aux principes du droit international si l'une des entités affirme démocratiquement sa volonté de se séparer de la "mère patrie». L'éclatement pourrait provenir de la fragile Fédération et entraîner à sa suite la République Srpska vers la RFY. R. Karadzic, l'ancien chef des serbes de Bosnie, n'affirmait-il pas que la séparation de la Bosnie en deux entités «ouvre la possibilité d'une séparation pacifique à l'instar des pays tchèque et slovaque, car notre objectif reste celui d'un rattachement à la Serbie»(49). Le principe de l'uti possidetis tel

(46) K. Zubak (croate) démissionnera (provisoirement) le 4 décembre 1995 pour marquer son désaccord avec l'attribution de la vallée de la Save aux serbes de Bosnie dans l'accord de paix.

(47) Le maire de Mostar était l'allemand H. Koschnik (ancien maire de Brême) jusqu'à son remplacement par l'espagnol $R$. Perez Casado (ancien maire de Valence) suite à la démission du premier découragé par l'attitude peu coopérative des croates.

(48) Voir Le Monde des 24-25 novembre 1995

(49) Voir Le Monde des 26-27 novembre 1995. 
qu'il est désormais envisagé (50) peut faciliter une telle issue faisant de la Bosnie un Etat représentant environ $30 \%$ de sa superficie originelle avec une "viabilité" territoriale à peine supérieure à l'actuelle découpage de l'Etat palestinien. Il ne s'agit pas d'un "scénario-catastrophe" mais bien d'une possibilité que l'accord de paix laisse entrevoir à moyen terme.

\section{II \\ LE DÉBUT DE LA PAIX : LES ASPECTS CIVILS DU REGLEMENT DE PAIX}

Les aspects civils du Règlement de paix constituent le deuxième grand volet de l'accord de paix. Cette partie est plus "ambitieuse" en ce qu'elle souhaite faire de la Bosnie un véritable Etat indépendant et souverain et qu'elle s'inscrit dans la durée contrairement aux aspects militaires dont le but immédiat et essentiel est de faire cesser la guerre.

Il fut tout d'abord nécessaire de prévoir la mise en œuvre de ces aspects civils et d'envisager les institutions aptes à reconstruire la Bosnie. A partir de ces prémices, l'accord de paix comporte trois grands axes : la restauration de l'Etat de droit, la paix civile (retour des réfugiés et des personnes déplacées) et la reconstruction matérielle du pays.

\section{A. La mise en œuvre des aspects civils du Règlement de paix}

L'annexe 10 de l'accord de paix est spécifiquement consacré à la mise en œuvre civile du Règlement de paix et s'appuie sur une conférence internationale pour sa concrétisation. L'annexe 11 sur la Force de police internationale qui vient le compléter s'appuie sur une mise en œuvre à l'aide de résolutions du Conseil de sécurité des Nations Unies. Des lettres jointes à l'accord complètent ce processus.

La mise en œuvre civile du Règlement de paix est ambitieuse par les domaines touchés et les institutions sollicitées. Sans que celles-ci soient expressément citées, l'article I de l'annexe 10 indique "qu'un nombre considérable d'organisations et d'institutions internationales sera sollicité à cet effet". Il est vrai que le programme implique des tâches très variées faisant appel à de multiples compétences (51). La nécessité de coordonner ces tâches fait l'objet d'une attention minutieuse puisque de nombreux points de l'annexe 10 sont consacrés à la nomination et au mandat d'un "Haut Représentant". Celui-ci sera nommé et, "conformément à une résolution du Conseil de sécurité», il devra mener à bien diverses missions : surveiller la mise en

(50) Voir notre article (avec R. MendI), AFDI 1994, pp. 11-40. S. Hille résume la principale contradiction entre le droit des peuples à disposer d'eux-mêmes et la reconnaissance de droits aux minorités: "This seems to be contradictory : either a minority has the right to self determination which means that it does not constitute a minority any more, or it is denied this right in the interest of the integrity of states, and in order to hinder separatist activities ", dans : "Mutual Recognition of Croatia and Serbia (and Montenegro)", Journal Européen de Droit International, $4 / 1995$, p. 604.

(51) L'alinéa $1^{\text {er }}$ de l'article I cite notamment: l'aide humanitaire, la réhabilitation des infrastructures et la reconstruction économique, la mise en place des institutions politiques et constitutionnelles, la promotion des droits de l'homme, le retour des personnes déplacées et des réfugiés et des élections démocratiques. 
œuvre du Règlement de paix, prendre des contacts avec toutes les parties impliquées, coordonner le travail des institutions et organisations, rendre compte aux Nations Unies et à l'Union européenne. En outre, il présidera la Commission Civile Mixte (CCM) mise en place pour réaliser ces différentes phases (article II). Cette Commission regroupera les Parties, le commandant de l'IFOR ainsi que les représentants des institutions et organisations intéressées. Réciproquement, le Haut Représentant participera à la Commission Militaire Mixte instituée par l'annexe 1A. Néanmoins, les barrières semblent étanches entre les aspects civils et militaires car l'annexe 10 prend soin de préciser que : «Le Haut Représentant n'aura aucune autorité sur l'IFOR et ne devra pas s'ingérer, d'une quelconque manière, dans la conduite des opérations militaires ou dans la chaîne de commandement de l'IFOR". La marque de la querelle entre les Etats-Unis et l'Union européenne est retranscrite dans ce bref passage. L'un privilégie le volet militaire, l'autre préfere envisager une reconstruction à long terme bénéfique à la stabilité en Europe.

C'est la conférence de Londres des 8 et 9 décembre 1995 qui va mettre en place les différents intervenants dans les aspects civils de la paix. Cette conférence réunit 42 Etats et 10 organisations internationales (52) et va désigner le Haut Représentant qui sera nommé par une résolution ultérieure du Conseil de sécurité : il s'agit du suédois Carl Bildt qui était déjà le médiateur de l'Union européenne. La résolution 1031 du 15 décembre 1995 approuve la mise en place d'un Haut Représentant et "agrée » la nomination de C. Bildt. La conférence va également créer un "Conseil de mise en application de la paix» qui remplace la Conférence internationale sur l'exYougoslavie créée en 1992, et qui comprendra un Comité de direction de 12 membres (53). Les conclusions de la Conférence insistent sur le lien entre le respect des droits de l'homme et le financement de la reconstruction ainsi que la nécessaire mobilisation d'un grand nombre d'organisations internationales. Cette conférence représente la "vision européenne" de l'accord de paix et selon un partage tacite il lui revenait de désigner le responsable de la mise en œuvre de ces aspects civils malgré la méfiance des Etats-Unis envers ce type de processus en général et envers C. Bildt en particulier. A plusieurs reprises, l'Union européenne ne manquera pas de soutenir ce processus en indiquant notamment que l'Union reste le plus gros contributeur en matière d'aide à la Bosnie (54) et qu'il lui revenait de désigner le Haut Représentant qui "devra être choisi en son sein"(55).

L'annexe 11 vient directement compléter l'annexe 10 en prévoyant la création, par le Conseil de sécurité des Nations Unies, d'une Force de police internationale (IPTF) des Nations Unies sous la forme d'une opération

(52) Les conclusions de la conférence sont dans le numéro hors série des $D A I$, op. cit., pp. $17-21$

(53) On y retrouve les représentants du G7, la Russie, le Président de la Commission européenne, le président en exercice de l'Union européenne, le représentant de l'Organisation de la Conférence Islamique et $\mathrm{C}$. Bildt.

(54) Dans «Les lignes directrices du Conseil des Affaires générales concernant l'ex-Yougoslavie" (Bruxelles, le 30 octobre 1995), l'Union européenne indique qu'elle a versé 1,6 milliards d'ECU au titre de l'aide humanitaire depuis le début du conflit. Texte dans le numéro hors série des DAI, op. cit., pp. 7-9.

(55) Conclusions du Conseil des Affaires générales sur les accords concernant l'ex-Yougoslavie mis au point à Dayton et signés à Paris, Bruxelles le 4 décembre 1995, ibid., pp. 14-15. Voir également : l'Action commune adoptée par le Conseil sur la participation de l'Union européenne à la mise en œuvre et au financement des aspects civils de l'accord de paix, adoptée sur la base de l'article J3 du traité sur l'Union européenne, Bruxelles le 11 décembre 1995, ibid., pp. 21. 
UNCIVPOL. Sa mission sera de surveiller, observer et contrôler les installations et les activités de police, de conseiller les forces et le personnel de police, de former les personnels de police et d'évaluer les menaces et l'aptitude de la police face à celles-ci (article III). Cette force pourra se déployer sur l'ensemble du territoire de la Bosnie.

C'est la résolution 1035 du Conseil de sécurité des Nations Unies du 21 décembre 1995 qui va créer cette Force de police civile, dénommée Groupe internationale de policc (GIP), pour un an à compter du transfert d'autorité et qui sera chargée des missions prévues à l'annexe 11. Cette force ainsi que le Bureau civil des Nations Unies seront "sous l'autorité du Secrétaire général " en liaison avec le Haut Représentant. Il est prévu que cette Force comportera 1700 "moniteurs" provenant de différents pays. Le recrutement de cette Force s'achèvera théoriquement le 15 janvier 1996 avec des policiers issus de 28 Etats et la nomination de l'irlandais P. Fitzgerald à leur tête. Néanmoins, la lenteur de la constitution de cette Force ainsi qu'une liaison mal définie avec l'IFOR vont créer des problèmes lors de l'enlèvement de 16 civils bosniaques à Sarajevo le 2 janvier 1996, chacun se renvoyant la responsabilité de la garantie de la liberté de mouvement. De même, le sous effectif de cette Force (un quart des policiers prévus était opérationnel fin février) empêchera de contrôler efficacement la réunification de Sarajevo dont les quartiers serbes (Vogosha, Ilidza ou Grbavica) vont se vider dans la plus totale anarchie sans que l'IFOR et la Force de police se mettent d'accord sur leurs missions respectives dans ce cadre. Visiblement, l'IFOR se désintéresse des aspects civils de l'accord et si, à la date du 19 mars, le bilan des aspects militaires est plutôt positif par rapport à l'échéancier de l'accord de paix, en revanche, le bilan des aspects civils est beaucoup moins satisfaisant. Il est vrai que ces derniers aspects doivent s'envisager dans la durée et que l'échéancier n'a pas la précision des aspects militaires.

\section{B. L'Etat de droit}

La restauration de l'Etat de droit est l'un des objectifs de l'accord de paix. S'il n'a pas retenu l'attention immétiate des commentateurs, c'est qu'il constitue un objectif à long terme contrairement à la fin du conflit armé. C'est pourtant à la lumière de la réalisation de cette partie de l'accord que l'on pourra juger de son succès ou de son échec. Il est trop tôt pour pouvoir se prononcer sur un bilan de cet aspect mais les modalités inscrites dans les annexes concernant la restauration de l'Etat de droit permettent d'envisager quelques perspectives qu'il serait bien hâtif de qualifier d'optimistes. Les annexes 3 et 4 consacrées aux élections et à la constitution sont le cour du processus de restauration de l'Etat de droit dont les ramifications s'étendent au-delà des simples aspects institutionnels.

L'annexe 4 de l'accord de paix est consacré entièrement à la constitution de la Bosnie-Herzégovine. Formée d'un Préambule suivi de 12 articles et deux annexes, la constitution de la Bosnie laisse une étrange impression de texte circonstancié et inachevé. Certains points jugés sensibles sont très détaillés mais d'autres sont beaucoup plus succincts alors que des oublis doivent être déplorés.

Le Préambule affiche un clair soucis de réconciliation dans le cadre d'une société pluraliste en affirmant son attachement à "la justice, la tolérance et la réconciliation". Les références au droit international en général (et notamment à ses principaux textes) et au droit humanitaire en particulier 
sont nombreuses. La physionomie économique de l'Etat sera résolument libérale car l'attachement à la propriété privée ainsi qu'à l'économie de marché est rappelé. Mais la formule qui clôt le préambule en résume toute l'ambiguïté : "Les Bosniaques, les Croates et les Serbes, en leur qualité de peuples constituants (avec d'autres) et de citoyens de la Bosnie-Herzégovine décident...". Le statut de "peuples constituants " coexiste avec la citoyenneté amenant une distinction entre un élément objectif (l'appartenance à un Etat) et un élément subjectif (l'appartenance à une "nationalité " ethnico-culturelle). De plus, la Bosnie n'est pas seulement peuplé de "bosniaques " (éventuellement distingués selon leur région d'origine) mais aussi de croates et de serbes. Cet aspect sémantique est fondamental car il tend à assimiler les seuls musulmans aux bosniaques et crée une confusion volontaire qui pourrait se révéler fatale pour l'avenir de cet Etat « unitaire $*$.(56).

L'article $1^{\mathrm{er}}$ est tout simplement consacré à la définition...de la Bosnie.) Il y est affirmé qu'il s'agit d'un Etat au sens du droit international, membre des Nations Unies, aux frontières reconnues, ayant pour capitale Sarajevo et «régi par la primauté du droit». Les dispositions les plus intéressantes résident dans l'alinéa 7 sur la citoyenneté: il existe une citoyenneté de Bosnie-Herzégovine et une citoyenneté de chaque entité. Une double citoyenneté est par conséquent possible et la «deuxième" citoyenneté peut provenir (selon l'alinéa 7, d) d'un autre Etat sous réserve d'un accord bilatéral. Les possibilités sont donc multiples et reflètent le caractère incertain de la "première" citoyenneté. Ces dispositions délicates sont entourées d'un luxe de précisions et de toutes les garanties concernant les droits de l'homme et les libertés fondamentales. Tous les grands textes concernant les droits de l'homme sont cités, soit directement dans l'article II, soit par renvoi à l'annexe I de la constitution qui énumère les quinze principales conventions en la matière (Prévention et répression du crime de génocide, Pactes de 1966...) auxquels la Bosnie est ou deviendra partie. Qu'il nous soit permis, sans ironie excessive, de remarquer que cette invocation massive est le plus souvent inversement proportionnelle à son application effective. Sans doute faut-il y voir une forme de protection, de conjuration contre un passé récent où ces textes étaient ignorés. L'alinéa 5 de l'article II méritera à lui seul une attention particulière: "Tous les réfugiés et personnes déplacées ont le droit de rentrer librement dans leur foyer d'origine" (cf. infra).

Le partage de compétences entre la Bosnie et les entités fait l'objet d'une description minutieuse. On y trouve la trace d'un partage de type fédéral (57) dans un Etat qui n'est pas une fédération mais la réunion d'une fédération (qui a déjà donné lieu à un partage des compétences avec le pouvoir central dans l'accord du 10 novembre 1995) et d'une République unitaire. On est en présence d'une construction à notre connaissance unique d'autant que «les entités ont le droit d'établir des relations bilatérales spéciales avec les Etats voisins dans le respect de la souveraineté et de l'intégrité territoriale de la Bosnie-Herzégovine" (article III, alinéa 2, a). De la même manière, chaque entité peut conclure des accords avec des Etats et des organisations inter-

(56) Les musulmans sont désormais appelés "bosnjaki " (boschniaks), mot distinct de «bosnjanci » (bosniaques) qui peut être appliqué aux autres citoyens (en anglais bosniaks et bosnians). Voir, C. SAMARY : "Mouvante identité des musulmans", dans Manière de voir $\mathbf{n}^{\circ}$ 29, op. cit., pp. 51-54.

(57) La Bosnie est responsable de la politique étrangère, du commerce extérieur, de la politique douanière, de la politique monétaire, de la politique d'immigration et des moyens de communication (liste non exhaustive). 
nationales avec le consentement de l'Assemblée parlementaire (sauf pour certains accords). Ces dispositions, indispensables au moment des négociations, doivent être envisagées en liaison avec les dispositions territoriales (cf. supra) et montrent le danger d'éclatement de l'Etat bosniaque.

Les principales institutions (Assemblée parlementaire, Présidence et Cour constitutionnelle) font l'objet des articles suivants. L'Assemblée doit comprendre deux chambres: la Chambre des peuples et la Chambre des représentants. La Chambre des peuples sera composée de 15 délégués (5 croates et 5 bosniaques provenant de la Fédération et 5 serbes de la république Srpska). La Chambre des représentants sera composée de 42 membres selon la proportion un tiers (serbes), deux tiers (Fédération). Les procédures sont organisées de manière à permettre un équilibre entre les trois composantes avec une possibilité tacite de veto lorsque le quorum «tripartite" n'est pas atteint.

C'est le même souci que l'on retrouve pour l'organisation de la Présidence. L'article $\mathrm{V}$ décrit la Présidence comme un organe collégial de trois membres élus au suffrage direct et correspondant aux trois composantes dont l'un sera désigné Président. Le consensus est privilégié mais les blocages sont envisagés ( $\mathrm{si}$ un membre considère qu'une décision est contraire à un intérêt vital de l'entité à laquelle il appartient). Les principaux pouvoirs de la Présidence sont: la politique étrangère, la demande d'adhésion de la Bosnie aux organisations internationales, la négociation, la "dénonciation" (expressément prévue) et la ratification (après l'approbation par l'Assemblée) des traités, la proposition du budget et l'exécution des décisions de l'Assemblée parlementaire. La Présidence est flanquée d'un gouvernement (dont on notera qu'il ne constitue qu'un alinéa dans l'article consacré à la Présidence). Le Président du Conseil des ministres est nommé par la Présidence mais doit être investi par la Chambre des représentants (également vrai pour les ministres). La nomination des ministres fait appel à un subtil équilibre puisque deux tiers au plus doivent être issus de la Fédération et ils seront épaulés par des vice-ministres qui ne doivent pas appartenir au même "peuple" que le ministre. Le gouvernement est responsable de l'exécution des politiques et sa démission est obligatoire à la suite d'une motion de défiance de l'Assemblée parlementaire. L'article $\mathrm{V}$ reflète un régime globalement parlementaire mâtiné de présidentialisme sans qu'il s'identifie à un type précis. L'essentiel réside surtout dans l'équilibre entre les trois composantes qui conditionne le reste.

Une Cour constitutionnelle vient compléter ce dispositif. Celle-ci est composée de 9 membres dont 6 sont choisis par les entités et 3 par le Président de la Cour européenne des droits de l'Homme. Ces derniers ne peuvent être citoyens ni de Bosnie ni "d'un Etat voisin" (article VI). Hormis la première Cour qui comportera des particularités, les juges conservent leur mandat jusqu'à l'âge de 70 ans. Cettc Cour est compétente pour tout litige découlant de la constitution entre les entités. Par exemple, c'est elle qui devra "juger" si la décision d'établir des relations spéciales avec un Etat voisin est conforme à la constitution. Elle exercera également un contrôle de conformité des lois à la constitution et la Cour servira de juridiction d'appel pour les décisions de tous les tribunaux de Bosnie (58).

(58) Précisons également que sa saisine sera ouverte aux membres de la Présidence, au Président du conseil des ministres, au Président ou vice-Président des Chambres ainsi qu'au quart de chacune des deux Chambres de l'Assemblée parlementaire et des chambres des entités. 
Les autres dispositions constitutionnelles semblent de moindre importance (59) et l'on peut même être surpris par la présence d'un article complet (article VIII) consacré à la Banque centrale dans une constitution aussi minimaliste. On peut retenir de cette Banque centrale que son premier gouverneur sera nommé par le FMI et qu'il ne doit pas être citoyen de Bosnie ni d'un Etat voisin comme les membres de la Cour constitutionnelle nommés par le Président de la Cour européenne des droits de l'Homme. La composition du Conseil de direction de la banque reflète une nouvelle fois l'équilibre entre les entités.

Globalement, la constitution prévoit l'intervention de nombreuses organisations internationales dans sa phase transitoire (OSCE, ONU, Conseil de l'Europe, FMI, Union européenne...) par souci de neutralité (les dispositions transitoires sont précisées dans l'annexe II à la constitution).

On peut constater que cette constitution est « un défi aux lois de l'équilibre»(60) qui tente de faire coexister dans un Etat: une République ayant sa propre constitution, son armée, sa police et son système d'éducation avec une Fédération où les deux composantes ont également leur propre système et leur armée!

Pour garantir le respect des institutions prévues à l'annexe 4, l'annexe 3 précise le régime des élections qui devront avoir lieu. L'OSCE est largement sollicitée pour assister la Bosnie dans cette phase, le document de la CSCE de Copenhague (1990) sur les élections libres et démocratiques servant de trame et étant intégré comme Supplément à l'annexe 3 (l'OSCE sera aidée dans cette tâche par la Mission de surveillance de la Commission européenne, ECMM). Le rôle de l'OSCE fait l'objet d'un article complet (article II) où on la charge de veiller au déroulement de l'ensemble des élections nécessaires (Présidence, Chambres de la Bosnie et des entités, Assemblées cantonales et gouvernements municipaux). Ces élections devront avoir lieu dans un délai de 6 à 9 mois après l'entrée en vigueur de l'accord avec l'aide d'une Commission Provisoire des élections qui rassemble le Haut Représentant, des membres de l'OSCE et des représentants des Parties. Ce délai montre la volonté de régler l'essentiel du sort de la Bosnie dans l'année à venir. Il est néanmoins peu probable qu'une vie politique équilibrée puisse s'instaurer dans un délai aussi court, ce qui laisse augurer d'une victoire probable des partis nationalistes au pouvoir dans les trois zones (SDS serbe, HDZ croate et SDA bosniaque).

Mais il faut sans doute faire une place à part à l'article IV de l'annexe 3 consacré à l'éligibilité qui est un chef d'œuvre d'ambiguïté où toutes les solutions sont prévues avec l'impression que les questions sont enchaînées au fur et à mesure de la progression du raisonnement sans que la conclusion confirme l'introduction... Après avoir rappelé que tout citoyen de Bosnie âgé de 18 ans et figurant au recensement de 1991 possède le droit de vote, l'article précise : «Tout citoyen ne résidant plus dans la municipalité dans laquelle il ou elle résidait en 1991 sera, en règle général, censé voter, soit en personne, soit par procuration, dans ladite municipalité, sous réserve qu'il soit établi que la personne concernée a effectivement été enregistrée dans ladite municipalité et que cela soit confirmé par la commission locale des

(59) Notons que la constitution peut être révisée à la majorité des deux tiers "des votants présents en personne" à la Chambre des représentants mais aucune révision ne peut intervenir sur les droits et libertés visés à l'article II.

(60) Selon l'expression utilisée par S. Dizdarevic dans Manière de voir, $\mathbf{n}^{\circ} 29$, op. cit., p. 50. 
élections, ainsi que par la Commission Provisoire des élections. Cependant, ce citoyen peut demander à la Commission d'enregistrer son vote en un autre lieu. L'exercice du droit de vote d'un réfugié sera interprété comme une confirmation de son intention de retourner en Bosnie-Herzégovine. Le retour des réfugiés devrait être engagé au plus tard le jour des élections, de manière à permettre au plus grand nombre de personnes de participer directement aux élections en Bosnie-Herzégovine. La Commission peut prévoir, dans les règles et règlements électoraux, le droit de vote des citoyens non enregistrés lors du recensement de 1991. "Il n'est pas certain que cet article facilite les élections mais il est le reflet de la question plus large des personnes déplacées et des réfugiés.

\section{La paix civile}

La question des réfugiés et des personnes déplacées est une des plus dramatiques du point de vue humanitaire de ce conflit. Plus de trois millions de personnes ont été déplacées à l'intérieur de l'ex-Yougoslavie et environ un million ont trouvé refuge à l'étranger (61). Le HCR en charge de ce dossier veut éviter à la fois un renvoi brutal des réfugiés par les pays d'accueil (Allemagne, Autriche, Suisse, Suède...) et la création de camps de réfugiés permanents de triste réputation. L'annexe 7 de l'accord de paix sur les réfugiés et les personnes déplacées tente de répondre à ces préoccupations en prescrivant des garanties pour les personnes ayant subi ce sort.

L'annexe 7 se décompose en deux parties distinctes. La première institue un régime de protection pour les réfugiés et les personnes déplacées avec l'affirmation très claire d'un droit au retour : "Tous les réfugiés et personnes déplacées ont le droit de retrouver librement leur domicile d'origine» (article I, alinéa 1). Ce retour doit s'effectuer "en toute sécurité » et il sera nécessaire de supprimer toutes les législations ou pratiques d'intimidation, de dissuasion et d'incitation à la haine. Les biens seront restitués ou une indemnisation aura lieu en guise de compensation. Le HCR doit établir un plan de rapatriement en accord avec les Etats ayant accueilli des réfugiés (article I, alinéa 5). Des conditions favorables au retour devront être mises en place et des garanties pour l'accès des organisations et institutions concernées (CICR, PNUD...) sont prévues.

La deuxième partie est consacrée à la Commission pour les personnes déplacées et les réfugiés créée par l'annexe. Cette Commission comprendra des représentants des entités et des membres nommés par le Président de la Cour européenne des droits de l'Homme (9 membres au total). Son rôle est d'identifier les propriétaires des biens immeubles, de les restituer ou de procéder à leur indemnisation. La procédure devant la Commission est minutieusement prévue et un fonds auprès de la Banque centrale sera ouvert et géré par la Commission.

Nul doute que cette Commission devrait être confrontée à un long travail. Mais il faudra auparavant convaincre les millions de personnes déplacées ou réfugiées qu'un retour "en toute sécurité" est possible. Or, cet objectif semble contradictoire avec la consécration d'un découpage territorial qui entérine lui-même l'épuration ethnique. Un bosniaque peut-il retrouver sa maison au milieu de la République Srpska? Seuls les quelques lieux où une

(61) Voir le bilan indiqué supra, note 13. 
coexistence des populations a continué pendant le conflit peuvent concrétiser cette volonté de retrouver une société multi-ethnique et pluriculturelle. 20000 serbes ont continué de vivre dans le Sarajevo bosniaque alors que les serbes assiégeaient la ville. Dans ce cadre, la compensation financière pourrait rencontrer un plus vif succès que le retour des personnes dans leur foyer qui, ne l'oublions pas, doit être "volontaire".

Cependant, le plan de rapatriement prévu dans l'annexe 7 fut adopté à Genève le 16 janvier 1996, sous l'égide du HCR, en présence de représentants de 40 Etats et d'une vingtaine d'organisations internationales. Ce plan porte sur le rapatriement de deux millions de réfugiés ou personnes déplacées en Bosnie. Il commencera au printemps 1996 et devrait s'achever deux ans plus tard selon l'évolution de la situation politique. Ce plan était réclamé de toute urgence par les Etats d'accueil dont certains avaient commencé à envisager le retour des réfugiés après la fin du conflit. En décembre 1995, l'Allemagne annonçait que les 350000 réfugiés de l'ex-Yougoslavie perdraient le droit de rester sur son sol à compter d'avril 1996 sauf s'ils bénéficient de l'asile politique. Grâce au plan adopté à Genève, le retour sera organisé de juillet 1996 à la mi-1997.

Pour que le retour soit réellement attractif, les personnes déplacées et les réfugiés devront retrouver des conditions de vie décentes. Cette paix civile, pour le moment très aléatoire, doit s'accompagner d'un programme de reconstruction qui donne à la Bosnie la possibilité de retrouver des infrastructures et une vie quotidienne plus sereine.

\section{La reconstruction}

Les infrastructures de la Bosnie étant quasiment totalement inutilisables, l'accord de paix consacre une annexe $\left(\mathrm{N}^{\circ} 9\right)$ à la création des entreprises publiques en Bosnie-Herzégovine pour la reconstruction de l'économie et des infrastructures. L'accord crée une Commission sur les entreprises publiques chargée d'étudier la création d'entreprises publiques pour l'exploitation des installations publiques communes (postes, communications...). Cette commission sera composée de 5 membres: deux pour la Fédération, un pour la République Srpska et deux nommés par le Président de la BERD. De nouveau s'effectue le processus de nomination par une personnalité extérieure provenant d'une organisation dont la compétence s'étend à l'annexe. Les besoins immédiats entraînent la création de "l'Entreprise de Transport de BosnieHerzégovine" (article II) chargée de l'installation des transports, des routes, des chemins de fer et des ports. Le personnel de cet entreprise devra être "représentatif des groupes ethniques". Cette entreprise servira de modèle pour d'autres Entreprises Publiques Mixtes (énergie, poste, télécommunications) (article III).

D'une manière complémentaire, l'annexe 8 crée une Commission pour la conservation des monuments nationaux qui doit se prononcer sur le classement de biens présentant "un intérêt culturel, historique, religieux ou ethnique comme monuments nationaux" (article IV). Cette fois, c'est le Directeur de l'UNESCO qui doit nommer, selon la même logique, deux membres (sur cinq) de la Commission. Il s'agit de "reconstruire» le patrimoine culturel de la Bosnie et de le préserver. Le conflit fut souvent propice à la destruction d'édifices (notamment religieux) qui étaient autant de symboles distinctifs des populations. 
Les annexes n'abordent guère la question cruciale du financement de la reconstruction. Ce financement est l'objet principal de la Conférence internationale sur la reconstruction de la Bosnie qui s'est réunie pour la première fois à Bruxelles les 20 et 21 décembre 1995 en présence de 50 Etats et de 20 organisations internationales dont la Banque mondiale et l'Union européenne qui jouent un rôle moteur dans ce domaine. Le principe du financement par tiers fut adopté (un tiers Europe, un tiers Etats-Unis et un tiers pour le Japon et les autres pays)(62). Cette Conférence s'est réunie de nouveau à Paris les 25 et 26 janvier 1996 et l'on a pu estimer le coût des dommages de guerre à 125 milliards de francs alors que 25 milliards de francs sont mobilisables sur une période de trois à quatre ans(63). Le financement risque, par conséquent, d'être un point délicat d'autant que l'intérêt suscité par la Bosnie pourrait s'émousser une fois la paix revenue. C'est la raison pour laquelle on cherche à faciliter l'intégration de la Bosnie dans des organismes financiers internationaux, ce qui lui permettrait de rejoindre les circuits plus "classiques" de l'aide au développement. La Bosnie est devenue membre du FMI le 20 décembre 1995 grâce à un prêt des Pays-Bas lui permettant de régler la fraction des arriérés dus par l'ex-Yougoslavie dont elle était débitrice. En échange, le FMI a consenti un achat de monnaie d'urgence de l'ordre de 45 millions de dollars(64). La Banque mondiale qui faisait de la Bosnie un "membre en succession" lui a permis, grâce à un plan spécifique, de payer ses arriérés au titre de l'ex-Yougoslavie pour devenir membre de l'organisation.

La levée de l'embargo économique touchant l'ex-Yougoslavie et certaines parties de la Bosnie en particulier(65) a permis de faire repartir une économie moribonde. Diverses lettres annexées à l'accord de paix envisageaient déjà les futurs liens entre les anciens ennemis. Par exemple, une union douanière entre la RFY et la Bosnie, une chambre de commerce mixte ou le développement d'échanges éducatifs sont envisagés dans une des lettres croisées entre la RFY et la Bosnie en date du 21 novembre 1995(66). Dans le même sens, par une série de trois accords du 11 mars 1996, la RFY et la Croatie rétablissaient entre ces Etats le réseau des transports et des télécommunications.

III

\section{LA GARANTIE DE LA PAIX : DROIT ET JUSTICE}

Nous avons pu noter l'importance des références au droit international en général et au droit humanitaire en particulier (notamment dans la constitution). Il n'est pas une annexe, quel que soit son objet, qui ne rappelle l'importance du cadre juridique à respecter. Il n'est pas une annexe non plus

(62) Seuls 518 millions de dollars furent dégagés pour le premier trimestre 1996 lors de cette réunion.

(63) De son côté, la Banque mondiale dispose de 600 millions de dollars pour un budget évalué à deux milliards de dollars pour la Bosnie en 1996.

(64) Voir la chronique des faits internationaux, $R G D I P 1996 / 1$, pp. 256 et s.

(65) Les sanctions imposées aux serbes de Bosnie ont été levées unilatéralement par la Russie le 23 février et par le Conseil de sécurité des Nations Unies le 26 février 1996 consécutivement au respect du volet militaire de l'accord de paix.

(66) Le texte de ces lettres se trouve dans le numéro hors série des DAI, op. cit., pp. 60-61. 
qui ne mentionne les obligations découlant de l'existence du Tribunal Pénal International (TPI) pour les crimes commis dans l'ex-Yougoslavie. Ce dernier ne bénéficie pas, comme d'autres sujets, d'un traitement particulier dans un accord séparé, mais sa présence est sans cesse rappelée au détour des obligations incombant aux Parties. Le droit comme cadre et la justice comme objectif sont sans doute les garants nécessaires - même si pas suffisants d'une paix durable en Bosnie.

\section{A. Le droit international comme garant}

La prégnance du droit international se manifeste tout au long de l'accord de paix sous différentes formes. Les droits de l'Homme et le droit humanitaire occupent une place privilégiée qui s'explique par les nécessaires garanties dans ces domaines après un conflit qui a bafoué les plus élémentaires règles de base. L'arbitrage est également un instrument très présent dans l'accord à travers des modalités particulières et par l'apport d'une annexe consacrée à ce mode pacifique de règlement des différends. Le droit des organisations internationales imprègne l'accord par la variété des organisations concernées et par la diversité des procédures utilisées. Néanmoins, l'accord de paix ne semble pas s'être préoccupé de la question de la succession d'Etats qui resurgit à l'occasion des prétentions de la RFY à être le principal, voire l'unique, successeur de la Yougoslavie.

L'annexe 6 de l'accord de paix est consacrée uniquement aux droits de l'Homme et concerne la Bosnie-Herzégovine, la Fédération et la République Srpska. Par cet accord, les Parties s'engagent à respecter un ensemble de libertés et de droits fondamentaux et notamment une liste de droits provenant de la Convention européenne des droits de l'Homme (67). Une annexe à l'accord donne la liste des 16 principaux textes en matière de droits de l'Homme et de droit humanitaire que les Parties s'engagent à respecter (cette liste est la même que celle de l'annexe I qui suit l'accord sur la constitution). Mais l'intérêt particulier de l'annexe 6 réside dans la création d'une Commission des droits de l'Homme composée d'un médiateur (Ombudsman) et d'une Chambre des droits de l'Homme. Cette Commission est chargée d'instruire tous les cas de violation des droits de l'Homme et tout individu peut saisir la Commission. Le médiateur constitue l'élément central de ce dispositif. Il est nommé pour cinq ans par le Président de l'OSCE et ne peut être citoyen de Bosnie ou d'un Etat voisin (après cette période transitoire, cette Commission dépendra de la Bosnie). Ce médiateur a des pouvoirs juridictionnels en liaison avec la Chambre (68) qui est composée pour moitié de citoyens de Bosnie (4 de la Fédération et 3 de la République Srpska) et pour moitié de membres nommés par le Comité ministériel du Conseil de l'Europe $(7 \mathrm{mem}$ bres qui ne sont ni citoyens de Bosnie ni d'un Etat voisin). Si un règlement amiable est prévu (article IX), le déroulement des débats devant les Chambres (article $\mathrm{X}$ ) ne laisse aucun doute sur la nature juridictionnelle de la procédure qui comporte des phases écrites et orales en audience publique

(67) Le droit à la vie, la liberté de pensée, de conscience et de religion, le droit de fonder une famille, le droit de propriété, le droit à l'éducation et le droit à la liberté de déplacement et de résidence (article I).

(68) L'article V intitulé "Juridiction du médiateur" décrit le système complexe et détaillé de saisine de la Commission des droits de l'Homme. L'article VIII est consacré à la « Juridiction de la Chambre" et décrit la saisine possible par le médiateur ou directement par une partie ou par un individu. 
(selon l'article XII, la Chambre peut adopter des Règlements et Règles de procédure). La Chambre peut adopter des mesures provisoires et prend des décisions définitives et sans appel (sauf exception) qui ne seront pas suivies d'opinions individuelles. Les parties sont tenues d'exécuter intégralement les décisions.

Le dispositif mis en place par l'annexe 6 comporte toutes les garanties d'une véritable juridiction en matière de droits de l'Homme. Son travail se fera en collaboration et sous la surveillance des organisations et ONG dont le champ de compétence s'étend aux droits de l'Homme. E. Rehm, le rapporteur spécial de l'ONU sur les droits de l'Homme dans l'ex-Yougoslavie, sera probablement attentive au bon déroulement de la procédure. Il reste à en connaître le fonctionnement et l'impact, ce qui n'est pas possible pour le moment, cette Commission n'ayant rendu aucune décision.

Un bref accord sur l'arbitrage (annexe 5) fait de ce mode de règlement pacifique des différends celui qui sera privilégié pour résoudre tous les litiges survenant entre les entités (Fédération et République Srpska). Celles-ci s'engagent à y recourir pour tout différend à venir. Il est significatif de trouver un mode de règlement qui s'applique plutôt aux différends interétatiques (même si ce n'est pas exclusif) pour des entités à l'intérieur d'un même Etat. Cela renforce la configuration «internationale" des relations entre les composantes de la Bosnie. Néanmoins, on ne peut que saluer cette volonté de promouvoir les règlements pacifiques des différends que les Nations Unies cherchent à imposer pour tous les différends potentiels dans l'ex-Yougoslavie. La résolution 1038 du 15 janvier 1996 vient le rappeler à propos d'un différend entre la RFY et la Croatie sur la péninsule de Prevlaka qui est démilitarisée depuis un accord du 30 septembre 1992.

L'accord de paix est sans doute très significatif de la place que prennent désormais les organisations internationales sur la scène internationale. C'est une véritable cascade d'organisations qui apparaît à travers l'accord. Celles-ci n'ont jamais été aussi présentes dans un règlement de paix, ce qui confirme un processus de "fonctionnalisation» croissant où les organisations agissent à la place des Etats dans leur domaine de compétence. L'ONU, l'OSCE, le Conseil de l'Europe, l'Union européenne, le FMI, la Banque mondiale, l'UNESCO, la BERD... ainsi que des institutions internationales (HCR, PNUD, CICR...) sont présents (parfois seulement pour un modeste pouvoir de nomination) dans l'accord de paix. Certes, l'ONU vient légitimer les décisions prises et exerce des pouvoirs en fonction de l'accord, ce qui est un processus connu, mais l'importance des organisations dépasse largement cette nécessaire légitimation par le Conseil de sécurité des Nations Unies. Il faut y voir également la reconnaissance d'efforts anonymes mais constants de la part d'organisations durant le conflit (69).

Les querelles qui resurgissent, à l'heure actuelle, à propos de la succession de la Yougoslavie permettent de découvrir un aspect qui ne fut pas abordé (ou presque) dans l'accord de paix. Il est vrai que les avis de la Commission d'arbitrage de la Conférence sur la paix en ex-Yougoslavie semblaient avoir réglé cette question. L'avis $n^{\circ} 8$ rappelait très clairement que "le processus de dissolution de la RSFY mentionné dans l'avis $\mathbf{n}^{0} 1 \mathrm{du}$ 29 novembre 1991 est arrivé à son terme et qu'il faut constater que la RSFY

(69) Rappelons que le HCR, par exemple, a assuré un pont humanitaire aérien pour Sarajevo du 2 juillet 1992 au 9 janvier 1996 (le plus long de l'histoire) en acheminant 160000 tonnes de vivres et de médicaments. 
n'existe plus»(70). L'avis $n^{\circ} 9$ abordait directement la question de la succession : "Il doit être mis un terme à la qualité de membre de la RSFY dans les organisations internationales conformément à leurs statuts respectifs et (...) aucun des Etats successeurs ne peut revendiquer en tant que tel et pour lui seul le bénéfice des droits détenus jusqu'alors par l'ancienne RSFY en sa qualité de membre". Ce passage a le mérite de la clarté. L'avis $n^{\circ} 10$ concluait : "La RFY (Serbie et Monténégro) apparaît comme un Etat nouveau qui ne saurait être considéré comme l'unique successeur de la RSFY"(71). L'Union européenne dans ses conclusions du Conseil des affaires générales (le 4 décembre 1995) continue d'adopter cette ligne de conduite. Le point 6 des conclusions indique qu'après la reconnaissance mutuelle envisagée à Paris le 14 décembre "... les Etats membres de l'Union européenne sont disposés à envisager la reconnaissance de la RFY comme l'un des Etats successeurs de l'ex-Yougoslavie, sur la base des directives approuvées par le Conseil le 16 décembre $1991 »(72)$.

Pourtant, l'évolution récente de la question laisse l'impression de concessions importantes par rapport à cette ligne de conduite. La RFY affiche sa prétention à être le «continuateur" principal, voire unique, de l'ex-Yougoslavie. En fait, il semble que cet Etat s'approprie le titre de continuateur politique de l'ex-Yougoslavie même s'il accepte un partage sur le volet économique (73). Une négociation prenant en compte le partage des biens immeubles et le partage des actifs (en fonction de la part de l'armée fédérale récupérée par la RFY) est en cours. La France semble appuyer la démarche de Belgrade puisqu'elle fut la première à nommer un ambassadeur en RFY fin février 1996. La France estime qu'un acte de reconnaissance formel n'est pas nécessaire puisqu'il y a toujours eu des relations diplomatiques entre les deux Etats. Ce débat n'est pas clos mais montre que la délicate question de la disparition de la Yougoslavie continue d'être négociée en marge de l'accord de paix. La nouveauté des situations apparues à la fín de la guerre froide a apporté des modifications assez sensibles à la prise en compte de la succession d'Etats par le droit international (74).

\section{B. La justice comme objectif}

Il y a obligation pour «toutes les Parties de coopérer aux enquêtes et poursuites pour crimes de guerre et autres violations du droit humanitaire international ». Tel est le libellé de l'article IX de l'accord-cadre général signé

(70) Avis du 4 juillet 1992, texte dans RGDIP 1993/2, p. 590.

(71) Ibid., pp. 584 et s. Les avis suivants (du 16 juillet et 13 août 1993) complétaient ces affirmations puisque l'avis $n^{\circ} 11$ précisait les dates auxquelles les Etats issus de l'ancienne Yougoslavie ont succédé à celle-ci (8 octobre 1991 pour la Slovénie et la Croatie, 17 novembre 1991 pour la Macédoine, 6 mars 1992 pour la Bosnie et 24 avril 1992 pour la RFY), l'avis no 12 et $\mathrm{n}^{\circ} 14$ donnaient des détails sur la succession d'Etats, l'avis $n^{\circ} 13$ traitait des conséquences d'éventuels dommages de guerre sur la succession d'Etats, et l'avis $n^{\circ} 15$ abordait la question de la succession de la Banque nationale yougoslave. Textes dans RGDIP 1993/4, pp. 1102 et s.

(72) Texte dans le numéro hors série des $D A I$, op. cit., p. 15.

(73) Les biens de l'ex-Yougoslavie sont estimés à 2 milliards de dollars en or et en devises et à 90 milliards de dollars de biens communs. En 1992, le FMI avait évalué le partage de la dette (environ 10 milliards de dollars) : $36,52 \%$ revenaient à la $\mathrm{RFY}, 28,49 \%$ à la Croatie, $16,39 \%$ à la Slovénie, $13,2 \%$ à la Bosnie et $5,4 \%$ à la Macédoine. Voir Le Monde du 10 février 1996.

(74) Voir à ce sujet les deux colloques du CEDIN : G. Burdeau et B. STERn (dir.) : Dissolution, continuation et succession en Europe de l'Est, Montchrestien, Paris, 1994, 406 p.; H. RUIz FABRI et P. Boniface (dir.): Succession d'Etats en Europe de l'Est et avenir de la sécurité en Europe, Montchrestien, Paris, 1995, 176 p. 
à Paris le 14 décembre 1995. A l'image de l'accord-cadre, les annexes qui suivent rappellent, sous différentes formes, l'obligation de coopérer avec le TPI ainsi que les contraintes que cela fait peser sur certains protagonistes du drame yougoslave.

L'annexe 1A indique que la Commission Militaire Mixte n'aura pas pour membres "des personnes actuellement mises en accusation par le Tribunal international pour l'ex-Yougoslavie, ou susceptibles de l'être" (article VIII, alinéa 3). De même, l'article IX consacré aux échanges de prisonniers précise que la détention sera maintenue pour toute personne soupçonnée de manière à permettre une consultation adéquate par les autorités du Tribunal. La constitution (annexe 4) comporte d'intéressantes liaisons avec le TPI. L'article II sur les droits de l'Homme rappelle la nécessaire coopération avec le Tribunal (cette obligation existe également dans l'annexe 6 - article XIII consacrée aux droits de l'Homme) mais c'est l'article IX sur les dispositions générales qui mérite de retenir notre attention : "Aucun individu purgeant une peine infligée par le Tribunal international de l'ex-Yougoslavie et aucune personne inculpée par le Tribunal et qui n'a pas obtempéré à un mandat de comparution devant le Tribunal ne peut faire acte de candidature ni exercer un mandat électif ou toute autre fonction publique sur le territoire de Bosnie-Herzégovine». Ainsi devrait se clore la carrière politique de R. Karadzic, le «chef» des serbes de Bosnie inculpé par le Tribunal.

L'annexe 7 fait allusion, par défaut, au TPI puisqu'il est prévu une amnistie pour les infractions de droit commun sauf pour les crimes de droit commun et les violations du droit humanitaire international conformément aux statuts du Tribunal international pour l'ex-Yougoslavie (article VI). A l'annexe 8 consacrée à la Commission pour la conservation des monuments nationaux, ce sont les personnes qui font l'objet d'une mise en accusation ou qui ont refusé de comparaître devant le Tribunal qui ne peuvent faire partie de la Commission. Enfin, la Force de police internationale créée par l'annexe 11 doit transmettre les informations qu'elle possède au Tribunal (article VI).

Le TPI «imprègne" l'ensemble de l'accord de paix sans qu'une partie spécifique lui soit consacrée. Ce «statut» est à la fois confortable car la réitération a des vertus bien connues en droit international, mais un risque de marginalisation existe, personne ne se sentant particulièrement concerné. Le TPI va, par conséquent, aborder la phase de l'accord de paix avec quelques incertitudes quant aux possibilités qui lui sont offertes.

La coopération entre le TPI et l'IFOR va donner lieu à quelques distorsions, la Force de maintien de la paix ne s'estimant pas liée par les requêtes du Tribunal. Effectivement, l'annexe 1A, malgré les dispositions susmentionnées n'impose pas une claire collaboration entre le TPI et l'IFOR. L'article VI consacré au déploiement de l'IFOR précise que la Force peut remplir des «tâches de soutien» dans la limite de sa tâche principale, mais parmi les missions annexes citées ne figure pas le soutien au TPI. Le soutien "politique»(75) ne manque pas mais la base juridique fait défaut. Faute d'exister,

(75) Le Président Clinton déclarait le 14 décembre 1995 qu'il faudra "demander des comptes à ceux qui sont accusés de crimes de guerre". Les différents textes de l'Union européenne ne manquent pas de rappeler l'impérative collaboration avec le TPI, tout comme les Groupes de Contact (voir: la Déclaration conjointe du Groupe de Contact international et du Groupe de Contact de l'Organisation de la Conférence islamique, Royaumont, le 13 décembre 1995, texte dans le numéro hors série des DAI, op. cit., p. 22). 
le contact entre les deux institutions va se nouer progressivement. Le procureur Goldstone se rend à Sarajevo le 22 janvier 1996 pour réclamer à l'amiral Smith une assistance et une protection pour les enquêteurs. Un accord (resté secret) a finalement été trouvé sous la forme d'un "code de bonne conduite" concernant l'arrestation de personnes soupçonnées de crimes de guerre. La Bosnie transmettra la liste des personnes soupçonnées au TPI qui devra confirmer avant toute arrestation que la personne incriminée est également suspecte à ses yeux. Dans ce cadre l'IFOR est prête à coopérer (76). Une liste diffusée auprès de l'IFOR et contenant 51 noms de personnes inculpées circulera à partir de la mi-février 1996 (quelques photos seront jointes à cette liste). A la même période, un communiqué du Conseil de l'Atlantique Nord réaffirme son soutien au TPI et indique que l'IFOR livrera les personnes «qui se trouvent en contact avec l'IFOR»(77). En réalité, l'IFOR va se charger plus largement de la protection des éléments de preuve, de la détention des criminels de guerre mis en accusation et de l'aide aux équipes d'enquêteurs.

Il est vrai que cette période coïncide avec la confirmation de la découverte de charniers, ce qui incite l'IFOR à une coopération accrue. Déjà le 21 décembre 1995 dans sa résolution 1034, le Conseil de sécurité, alerté par un rapport du Secrétaire général du 27 novembre, s'inquiétait de la protection des sites des charniers et exigeait "un accès immédiat et sans entrave " aux zones concernées ainsi que l'abstention par les serbes de Bosnie de toute action visant à "détruire, altérer, dissimuler ou détériorer tous les éléments de preuve"(78). Néanmoins, l'IFOR gardera une position plus "neutre" estimant que la garde des sites relève d'une tâche civile et policière et qu'elle peut simplement créer un "environnement sûr" (selon une déclaration du général Joulwan). Il est certain que la latitude laissée par plusieurs passages de l'annexe 1A, qui permettent au commandement de l'IFOR de prendre des mesures supplémentaires nécessaires à leurs missions, aurait autorisé une vision plus extensive de la coopération avec le TPI.

De cette coopération dépend la crédibilité du TPI et de l'IFOR. La provocation dont $R$. Karadzic et $R$. Mladic ont fait une arme le prouve. Le 16 novembre 1995 le TPI les mettait en accusation pour avoir été "directement et personnellement responsables des atrocités commises contre la population musulmane de Srebrenica». Eliminés de la vie politique et de toute fonction officielle par un tel acte, les deux dirigeants serbes n'en restaient pas moins libres. R. Karadzic ne pouvait plus dialoguer avec les autorités officielles et le Président du Parlement serbe de Pale, M. Krajisnik, devint l'interlocuteur de l'IFOR. Il en allait de même pour R. Mladic, le chef de l'armée des serbes de Bosnie, accusé de "génocide, crimes contre l'humanité et violations des lois et coutumes de la guerre». Le TPI ne connaissant pas le jugement par contumace, il ne pouvait que transformer les anciens dirigeants en fugitifs criminels sous le coup d'un mandat d'arrêt international (article $61 \mathrm{du}$ Statut du TPI). Depuis les provocations des deux hommes ne cessent de se multiplier sans que l'IFOR puisse, ou souhaite, intervenir (79).

(76) Le Président du TPI, A. Cassese a confirmé l'existence d'un tel accord tout en précisant que l'IFOR n'est pas une police judiciaire, dans Le Courrier international, $\mathbf{n}^{\circ} 280$ du 14-20 mars 1996.

(77) Communiqué du 14 février 1995, Revue de l'OTAN, n 2, mars 1996, p. 9.

(78) Une carte des principaux charniers repérés se trouve dans $L e M o n d e$ du 26 janvier 1996. Ceux de Srebrenica, Ljubija, Sanski Morst et Vocuza ont déjà été clairement identifiés.

(79) En février 1996, R. Karadzic a pu traverser le territoire de la Bosnie et passer quatre points de contrôle de l'IFOR sans être inquiété. 
En dépit de cet échec (provisoire?), les arrestations se sont poursuivies parmi les personnes soupçonnées. On peut signaler particulièrement celle, le 30 janvier, de deux officiers serbes arrêtés en Bosnie et transférés après enquête (80) au TPI à La Haye. Finalement, le $1^{\text {er }}$ mars 1996 , le général Djukic, adjoint du général Mladic, sera inculpé de crime de guerre, de crimes contre l'humanité et de violations des lois ou coutumes de la guerre. Ce dernier a participé au bombardement de cibles civiles à Sarajevo (81).

La Croatie n'a pas non plus prouvé de bonne volonté envers le TPI. L'adoption d'une législation permettant l'extradition de croates inculpés et la reconnaissance du statut du TPI furent de nombreuses fois retardées et des dirigeants inculpés se voyaient gratifiés de promotions au lieu d'être livrés à la justice. Finalement, le général T. Blaskic ainsi que le "vice-président" de la Herceg-Bosna, D. Kordic ont été démis de leur fonction après de multiples pressions. Le général Blaskic a fini par se rendre «volontairement» à La Haye où il fut la sixième personne incarcérée (dont certains sont détenus aux Pays-Bas, en Allemagne et en Autriche) sur une liste de 57 inculpés.

Sans que le bilan soit définitif, il est intéressant de constater que le TPI fonctionne correctement et qu'il ne cède pas aux pressions ou aux menaces de ceux qui souhaiteraient son inactivité ou sa disparition. Certes, le succès n'est pas assuré car de multiples problèmes se posent, à commencer par l'aspect financier (82) et les restrictions de son Statut(83). Mais le TPI a surmonté de nombreux handicaps grâce à l'obstination et la persévérance de ses membres dont le Président A. Cassese est le meilleur exemple. De simple alibi, tel qu'il apparaissait lors de sa création, il est devenu une juridiction active qui a réussi à ne pas disparaître comme monnaie d'échange lors de la négociation de l'accord de paix à Dayton. Il est maintenant parvenu à l'âge adulte et fonctionnera si les Parties à l'accord l'y aident et si le Conseil de sécurité lui en laisse le temps et les moyens (84). Dans ce sens, le Conseil de sécurité devra être conscient que le TPI représente la communauté internationale et non les seuls "Etats vainqueurs" comme à Nuremberg ou à Tokyo.

Il n'y a guère d'échelle dans l'ordre de l'horreur même si les serbes semblent responsables de la majorité des exactions, suivis par les croates et les bosniaques. La force du TPI sera de ne pas distinguer selon l'origine de ces actes. Mais son efficacité dépend de la bonne volonté des Etats, le TPI n'étant pas doté d'une police internationale. Les Etats de l'ex-Yougoslavie, les Etats occidentaux et l'IFOR se trouvent néanmoins dans l'obligation de mettre en œuvre une procédure dont certains pensaient qu'elle ne servirait guère.

(80) Le procureur avait réclamé le 7 février l'application de mesures conservatoires (article 40 du statut du Tribunal) pour éviter que le suspect n'échappe au Tribunal.

(81) Où 10000 civils furent tués (dont 1500 enfants) pendant les 44 mois de siège.

(82) Le budget prévisionnel du TPI pour 1996 est de 40,8 millions de dollars mais il dispose seulement de 7,6 millions jusqu'au 31 mars. On trouvera un intéressant tableau récapitulatif sur le TPI (budget, actions, handicaps...) dans Le Courrier international, op. cit.

(83) Le TPI n'est pas compétent pour les crimes contre la paix, ce qui peut paraître paradoxal pour un organe judiciaire créé sur la base d'une "menace contre la paix". Il lui sera difficile de juger les responsables de l'agression dont le Président de la RFY S. Milosevic.

(84) Lors de la première décision de la Chambre d'appel du TPI concernant le cas D. Tadic (compétence, celui-ci faisant partie du groupe responsable du camp d'Omarska), le TPI s'est livré à l'exercice périlleux de l'auto-justification de sa création par le Conseil de sécurité. Voir le commentaire de M. Sassoli dans la RGDIP 1996/1, pp. 101-133. 
Il n'est pas possible de dresser un bilan définitif de l'accord de paix au moment où celui-ci commence à produire ses effets. Il semble néanmoins que le bilan de l'application militaire soit plutôt positif évitant aux promoteurs du plan de paix de perpétuer la série de déconvenues qui faisait de l'ex-Yougoslavie «un cimetière de réputations»(85). On ne peut cependant occulter l'acceptation d'une situation, a priori inacceptable, via cet accord de paix. La Paix signée à Paris est avant tout l'expression d'un statu quo, celui du découpage en zones militaires ethniques qui privilégie le sang au sol. Il faudrait aller plus loin que les arrières pensées chères à $P$. Valéry pour remonter aux sources de l'accord de paix. La conception subjective de la nation l'a emporté, par souci de réalisme, face à un "vouloir vivre ensemble" qui n'existe plus (86) et dont l'objectif dans l'accord de paix ne comporte pas toutes les garanties de réussite. Les défenseurs d'une Bosnie multi-ethnique ne sont plus guère nombreux (87) alors qu'il s'agissait d'une situation objective avant le conflit.

Finalement, on peut constater que l'absence de guerre fut préférée à une vraie paix. Lors d'une réunion du Groupe de Contact sur l'ex-Yougoslavie, le ministre français des affaires étrangères s'insurgeait contre "une dérive qui fait passer de la logique de paix à la logique de séparation" (88). Cette dernière "logique" loin d'être l'aboutissement du processus de l'accord de paix, semble au contraire en être l'origine. Même si le constat est abrupt, on ne peut sans doute mieux résumer cette situation que P. Garde à propos de l'accord de paix : "Nous avons théoriquement un compromis entre l'unité et le partage, mais tout ce qui tend au partage est réel et tout ce qui tend à l'unité est fictif»(89).

Mars 1996

(85) Expression empruntée à P. HASSNER à propos des échecs successifs de la Communauté européenne (1991-1992), de l'ONU (1992-1993) puis de l'OTAN (1993-1994). Voir: «Une autre analyse de la guerre en ex-Yougoslavie" (entretien), Relations Internationales et Stratégiques, $\mathrm{n}^{\circ} 18$, été $1995, \mathrm{p} .33$.

(86) Et que l'écrivain croate $M$. KRLEZA résume superbement: "Que Dieu me garde de la culture croate et de l'héroïsme serbe", citée dans Le Monde du 29 février 1996.

(87) Le général bosniaque A. Dudakovic, à l'opposé d’A. Izetbegovic, y croit encore. L'ex-Premier ministre de Bosnie, $H$. Silajdzic, semble également vouloir développer un courant moins nationaliste que la ligne présidentielle.

(88) Le Monde du 26 mars 1996.

(89) Entretien dans Le Monde du 28 novembre 1995. 